Article

\title{
Evaluation of Yeast Derivative Products Developed as an Alternative to Lees: The Effect on the Polysaccharide, Phenolic and Volatile Content, and Colour and Astringency of Red Wines
}

\author{
Rubén Del Barrio-Galán ${ }^{1,2, *} \oplus$, Cristina Úbeda ${ }^{3}$, Mariona Gil $^{4}$, Marcela Medel-Marabolí ${ }^{1}$, \\ Nathalie Sieczkowski ${ }^{5}$ and Álvaro Peña-Neira ${ }^{1}$ \\ 1 Department of Agro-Industry and Enology, Faculty of Agronomical Sciences, University of Chile, \\ P.O. Box 1004, Santa Rosa 11315, La Pintana, Santiago, Chile; mmedel@uchile.cl (M.M.-M.); \\ apena@uchile.cl (Á.P.-N.) \\ 2 Lallemand Inc. Chile y Compañía Limitada, Rosario Norte 407, piso 6, Las Condes, Santiago, Chile \\ 3 Instituto de Ciencias Biomédicas, Facultad de Ciencias, Universidad Autónoma de Chile, \\ Santiago 7500912, Chile; c_ubeda@us.es \\ 4 Instituto de Ciencias Químicas Aplicadas, Inorganic Chemistry and Molecular Materials Center, \\ Universidad Autónoma de Chile, el Llano Subercaseaux 2801, San Miguel, Santiago, Chile; \\ marionagilicortiella@gmail.com \\ 5 Lallemand SAS, 19 rue des Briquetiers, BP 59, 31702 Blagnac, France; nsieczkowski@lallemand.com \\ * Correspondence: rdelbarriogalan@gmail.com; Tel.: +56-2-29785730
}

Academic Editors: David Jeffery and Paul Smith

Received: 11 February 2019; Accepted: 5 April 2019; Published: 15 April 2019

check for updates

\begin{abstract}
Due to the increase of the use of yeast derivatives (YDs) in winemaking to improve the technological and sensory properties in wines, in this work we evaluated the effect of the post-fermentation application of different yeast derivative products on the physical and chemical properties and astringency of red wines during two consecutive harvests. A commercial and two experimental new yeast derivatives were applied at a medium-high dosage $(30 \mathrm{~g} / \mathrm{hL})$. The addition of different yeast derivatives in red wine increased the concentration of different polysaccharide fractions and, therefore, the total polysaccharide content, producing a decrease in the duration of the wine astringency perception over time. The use of yeast derivatives could produce an adsorption/clarification and/or protective effect on the phenolic compounds. However, it did not produce an important modification of the colour parameters. An intensification or a lower decrease of the most volatile compound groups was produced, but it depended on the YDs and yeast strain used in fermentation and post-fermentation processes.
\end{abstract}

Keywords: yeast derivatives; polysaccharides; red wine colour; phenolic compounds; volatile compounds; astringency

\section{Introduction}

Yeast derivatives have been studied more and more in recent years due to the positive effects they can produce in wines during and after alcoholic fermentation. Their use is indicated during the winemaking process mainly to improve the technological (tartrate and protein stability) and sensorial characteristics and to remove some undesirable wine compounds [1]. These products have been proposed for some years as an alternative to traditional ageing via the lees technique, because they can provide the same benefits while avoiding or reducing some of the disadvantages (the release of polysaccharides during ageing on lees is too slow) [2]. 
Regarding the sensory quality of the wine, the improvements are mainly attributed to the mannoproteins released by the yeast derivatives during wine ageing [1,3-5]. In general, yeast derivatives are classified into five groups depending on the process used in the manufacture, the composition, and the degree of purification: inactivated dry yeasts, yeast autolysates, yeast cell walls, yeast extracts [1], and purified mannoproteins.

Generally, there are few commercial preparations based on yeast derivatives with a high degree of purification, mainly because it is a rather laborious and expensive process. For this reason, most of the commercial preparations available on the market for ooenological use are composed of specific inactivated dry yeast (SIDY), yeast autolysate (YA), or yeast cell walls (CW). SIDYs are products that are inactivated by different methods (enzymatic or thermal) and then subjected to a drying process. YAs are obtained after an incubation period of the yeast biomass, with controlled temperature, to favour the release of their own enzymes from the intracellular content and, subsequently, they are inactivated and dried. Finally, the CWs are obtained from YAs by a centrifugation process [1].

Generally, for ooenological applications, these yeast derivatives are selected for their particular characteristics, such as the high content of polysaccharides, mainly mannoproteins or other yeast compounds such as sterols, amino acids, and peptides. SIDYs are commonly used in winemaking as protectors during the rehydration of active dry yeast [6] and to improve the alcoholic [7] and malolactic fermentation [8]. In addition, several studies have described that the mannoproteins released from yeast derivatives may improve several taste characteristics of red wines such as volume and structure as well as decrease astringency and bitterness [9-11], and thus they are considered the polysaccharides from yeasts with the highest ooenological interest. This effect is mainly due to the interaction between the mannoproteins and the phenolic compounds of the wine, limiting the self-aggregation of the tannins [12], resulting in more stable polymeric structures that do not interact with the mouth salivary proteins and, therefore improving the sensory characteristics mentioned above and contributing in a remarkable way to contribute to wine mouthfeel properties [13]. Recent studies have shown the ability of YDs to adsorb or interact with the wine phenolic compounds, modifying their sensory characteristics $[14,15]$. The interaction between polysaccharides and phenolic compounds can also help stabilize the colour of wines due to the formation of more stable polymer pigments that can prevent or reduce the oxidation of wine, as mentioned in several studies [16,17]. Conversely, other studies conclude that this interaction produces a loss of colour in red wines $[4,9,11,18-20]$.

YDs can also modify the volatility of wine compounds, because they have the capacity to adsorb some wine volatile compounds $[3,21]$. On the other hand, the application of these products may induce an enrichment of new volatile compounds in wine, which are formed during their processing and could be released into the wines [22].

Astringency is considered a tactile sensation; some authors point out that this phenomenon could be due to a loss or alteration of oral cavity lubrication [23,24]. According to Bennick (2002) [25], the proteins secreted by the parotid gland seem to have the greatest capacity to bind to phenolic compounds. YDs' polysaccharides could have a lubricating effect and reduce this tactile sensation. Astringency is one of the most important characteristics that define the quality and persistence of red wine [26,27]. Astringency is a complex sensory characteristic, which depends on time and is related to several sensations that can be perceived simultaneously. The time intensity (TI) method has been used in wine to analyse the bitter and astringent sensations caused by phenols [28], the interaction between astringency and sweetness [29], and astringency characterization in commercial wines [30].

Therefore, the objective of this work was to evaluate the effect of the application of different YDs: one commercial SIDY and two experimental new YDs (YA and CW) on the physical and chemical properties and astringency of red wines previously fermented with two different yeasts, which produce different contents of polysaccharides during the fermentation process. The comparative study of polysaccharide release during the fermentation carried out by these yeasts has already been evaluated in another study carried out by our group [31]. For this reason, it is not the objective of this study to compare the two types of yeast. 


\section{Results and Discussion}

\subsection{Effect of YDs on the Polysaccharide Content}

Figure 1 shows the concentration of total polysaccharides and of the different fractions identified and quantified, according to their molecular weight, after the treatment period (2MT) and three months of bottle storage (3MB) in the 2015 harvest. Three different polysaccharide fractions were identified and quantified, and were classified according to their molecular weight: F1 corresponds with the polysaccharide fraction with a high molecular weight (1200-110 kDa); F2 corresponds with the polysaccharide fraction with a medium molecular weight (110-20 kDa); and F3 corresponds with the polysaccharide fraction with a low molecular weight $(20-5 \mathrm{kDa})$.

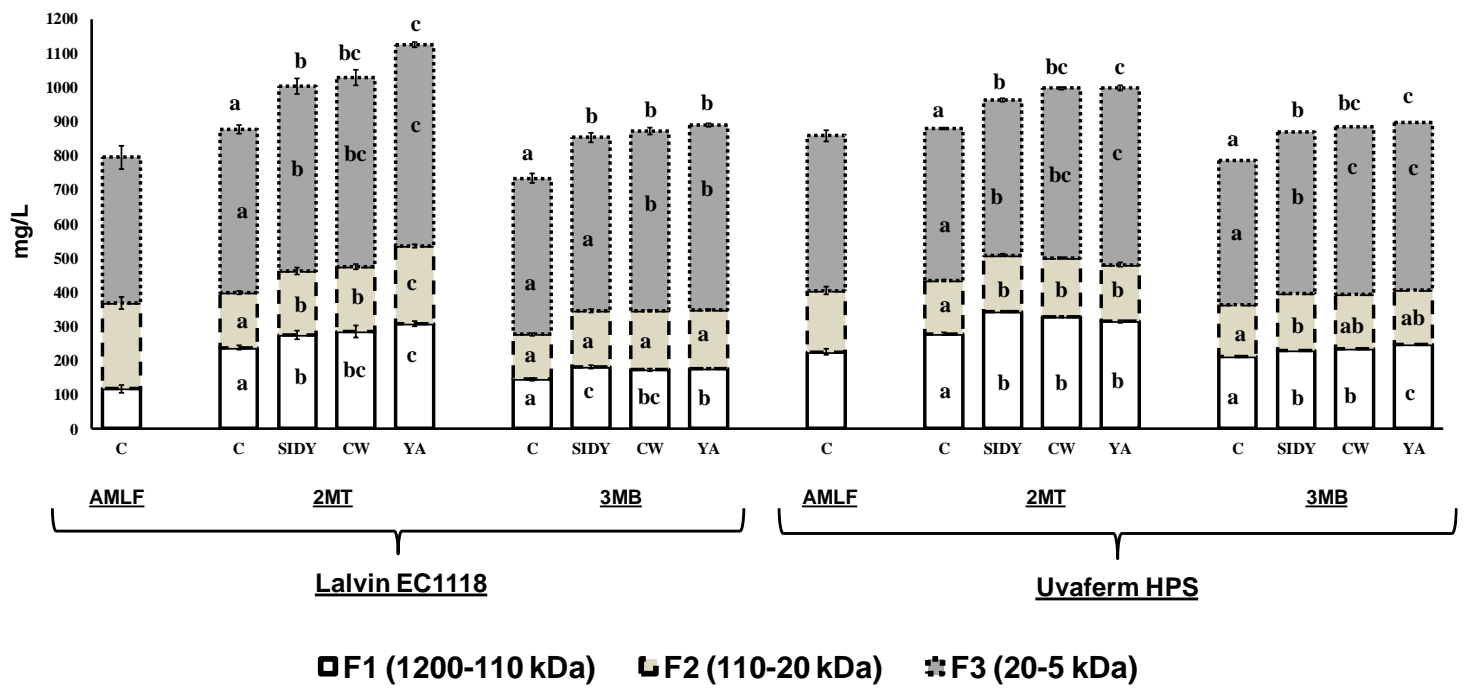

Figure 1. Polysaccharide fractions contents $(\mathrm{mg} / \mathrm{L} \pm \mathrm{SD})$ of the different red wines studied. Columns with different letters indicate statistically significant differences $(p<0.05)$ between the different treatments: AMLF (after malolactic fermentation); 2MT (two months of treatment); 3MB (three months of bottle storage).

In general, it was observed that wines treated with the different YDs had a higher concentration of all polysaccharide fractions and total polysaccharides than control wines after the $2 \mathrm{MT}$ and $3 \mathrm{MB}$ periods for both wines fermented with Lalvin EC1118 ${ }^{\circledR}$ and fermented with Uvaferm HPS ${ }^{\circledR}$ (both supplied by Lallemand-Sudamérica (Santiago de Chile, Chile). However, in general, insignificant differences were found between the different YDs studied and only in a few cases the wines treated with YA have a higher content of polysaccharides than the wines treated with SIDY and CW.

The low molecular weight polysaccharide fraction (F3) was the most abundant fraction in all the cases studied, revealing that, in general, all the wines treated with YDs had a higher content than the control wines, and those treated with YA had the highest content.

This effect of the increase of the wine polysaccharides by the addition of YDs, after alcoholic fermentation, has been reported in other studies carried out in red wines of other varieties [2,4,9], but these studies did not report data about the molecular weight of the different polysaccharide fractions.

In general, it was observed that the content of polysaccharides decreased between the 2MT and $3 \mathrm{MB}$ periods, probably due to the filtering process.

\subsection{Effect of YDs Application on the Phenolic Content}

Figure 2 shows the results obtained in the analysis of total polyphenols, tannins, and anthocyanins, and Table 1 shows the content of the different low molecular weight phenolic groups studied for both types of fermented wines (Lalvin EC1118 ${ }^{\circledR}$ and Uvaferm HPS ${ }^{\circledR}$ ). In general, it was observed that the content of the studied phenolic families decreased or remained stable throughout the study period. 


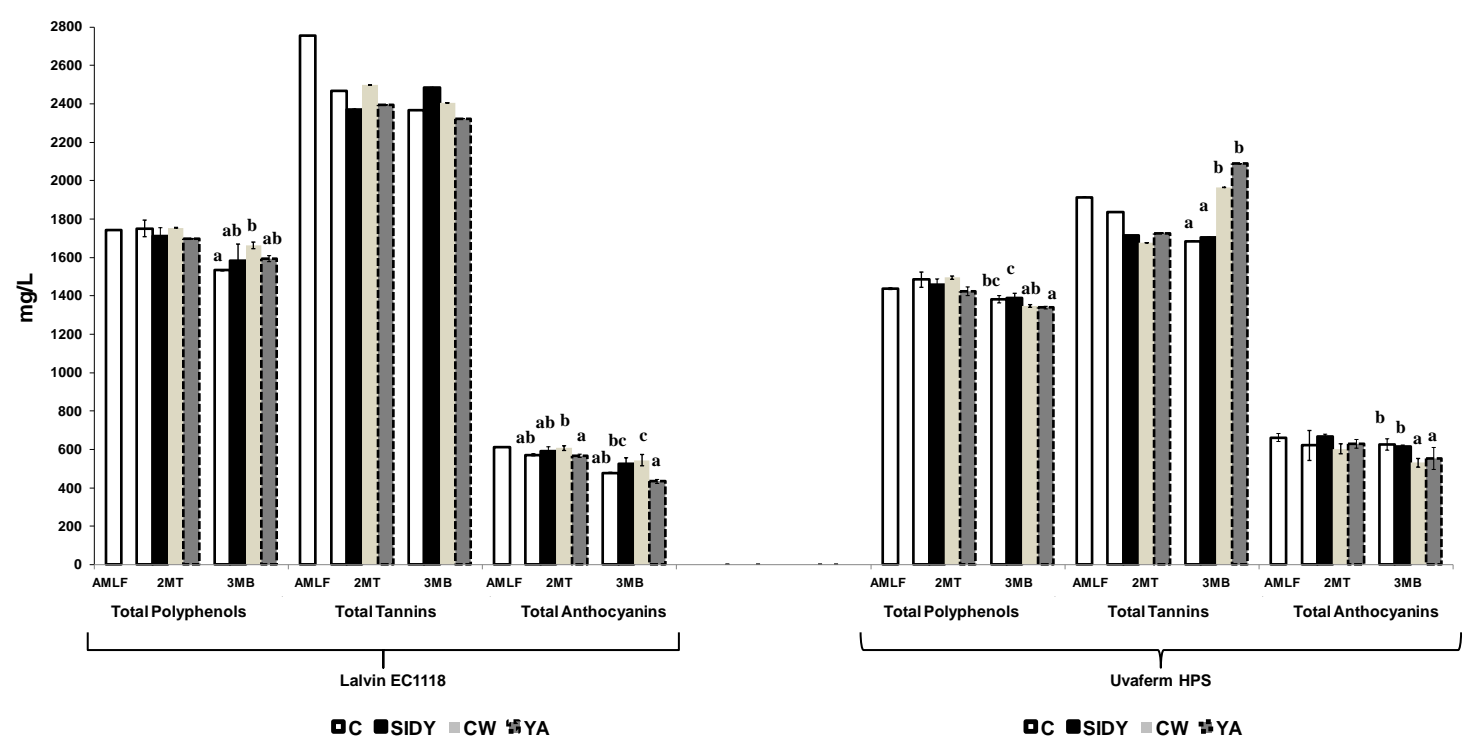

Figure 2. Total polyphenol, tannins, and anthocyanins concentration $(\mathrm{mg} / \mathrm{L} \pm \mathrm{SD})$ of the different wines studied. Columns with different letters indicate statistically significant differences $(p<0.05)$ between the different treatments: AMLF (after malolactic fermentation); 2MT (two months of treatment); $3 \mathrm{MB}$ (three months of bottle storage).

The application effect of the different YDs on the phenolic families depended on the type of YDs and the phenolic group studied. Thus, it was observed that the wines fermented with Lalvin EC1118 ${ }^{\circledR}$ and treated with the different YDs presented a similar or higher content of all the phenolic families studied after the $2 \mathrm{MT}$ and $3 \mathrm{MB}$ periods. In general, similar results were obtained in those wines fermented with Uvaferm HPS. However, it was observed that in some treatments carried out with YDs a decrease in certain phenolic families' content was also observed but there was no clear trend.

The trend of the low molecular weight phenolic groups was not clear during the study. Depending on the YDs used, the content was higher or lower than in the control wines. These results may be due to different effects brought out by the YDs: an adsorption effect of phenolic compounds by the polysaccharides released by the YDs, producing a clarification effect; and another protective effect of the phenolic compounds in the wines treated with these products, producing a greater degradation of phenolic compounds in the control wines.

Some studies carried out with different YDs in red wines indicated that their use could modify the phenolic composition, mainly due to the higher release of polysaccharides (mannoproteins) from yeast cell walls. However, the results obtained have been often contradictory, probably due to the different wine composition, as well as the different YDs composition and characteristics used. In some studies it was observed that treatment with YDs decreased the wine's phenolic content $[4,18,31,32]$. However, in other studies, the authors observed that the phenolic content of wines treated with YDs was similar to or slightly higher than the control wines [2,33]. In addition, in a study carried out by Watrelot et al. [34] it was observed that the exogenous addition of polysaccharides to Cabernet Sauvignon samples produced an increase in the tannin concentration. 
Table 1. CIELab parameters and low molecular weight phenolic compounds content $(\mathrm{mg} / \mathrm{L} \pm \mathrm{SD})$ of the different red wines studied.

\begin{tabular}{|c|c|c|c|c|c|c|c|c|}
\hline \multirow[b]{2}{*}{$2 \mathrm{MT}$} & \multicolumn{4}{|c|}{ Lalvin EC1118 ${ }^{\circledR}$} & \multicolumn{4}{|c|}{ Uvaferm HPS ${ }^{\circledR}$} \\
\hline & CONT & SIDY & CW & YA & CONT & SIDY & $\mathrm{CW}$ & YA \\
\hline CI & $11.3 \pm 0.07 b$ & $9.52 \pm 1.07 \mathrm{ab}$ & $9.15 \pm 1.11 \mathrm{a}$ & $10.6 \pm 0.06 \mathrm{ab}$ & $5.84 \pm 0.01 \mathrm{a}$ & $5.70 \pm 0.02 \mathrm{a}$ & $9.41 \pm 0.90 c$ & $7.49 \pm 0.41 b$ \\
\hline $\mathrm{L}^{*}$ & $49.3 \pm 0.23$ & $55.3 \pm 4.29$ & $56.8 \pm 4.48$ & $54.3 \pm 4.21$ & $71.0 \pm 0.29 c$ & $71.7 \pm 0.17 c$ & $56.0 \pm 2.71 \mathrm{a}$ & $63.3 \pm 0.81 b$ \\
\hline$a^{*}$ & $44.0 \pm 0.55$ & $38.9 \pm 3.60$ & $37.7 \pm 2.13$ & $44.1 \pm 2.00$ & $31.6 \pm 0.55 \mathrm{a}$ & $31.3 \pm 0.27 \mathrm{a}$ & $47.3 \pm 2.15 c$ & $40.1 \pm 0.49 b$ \\
\hline $\mathbf{b}^{*}$ & $9.18 \pm 0.24$ & $10.0 \pm 0.62$ & $10.5 \pm 1.68$ & $8.32 \pm 0.38$ & $13.3 \pm 2.15 b$ & $11.2 \pm 0.23 \mathrm{ab}$ & $8.4 \pm \mathrm{a} 0.63$ & $8.2 \pm 1.54 \mathrm{a}$ \\
\hline HBA & $52.2 \pm 0.42$ & $51.4 \pm 0.22$ & $53.1 \pm 0.54$ & $54.5 \pm 2.78$ & $44.8 \pm 0.07 a$ & $45.5 \pm 1.08 \mathrm{ab}$ & $45.9 \pm 0.82 \mathrm{ab}$ & $47.2 \pm 0.86 b$ \\
\hline HCA & $14.8 \pm 0.10$ & $14.6 \pm 0.20$ & $14.9 \pm 0.07$ & $15.2 \pm 0.72$ & $14.1 \pm 0.29$ & $14.0 \pm 1.21$ & $14.3 \pm 0.44$ & $14.9 \pm 0.60$ \\
\hline HCATE & $1.89 \pm 0.04$ & $1.84 \pm 0.07$ & $1.93 \pm 0.01$ & $2.09 \pm 0.19$ & $4.42 \pm 0.06$ & $4.48 \pm 0.02$ & $4.42 \pm 0.22$ & $4.64 \pm 0.12$ \\
\hline TFL & $43.2 \pm 0.20 \mathrm{a}$ & $44.3 \pm 0.59 \mathrm{ab}$ & $47.4 \pm 0.04 b$ & $45.1 \pm 2.51 \mathrm{ab}$ & $40.8 \pm 1.11 b$ & $42.3 \pm 1.02 \mathrm{~b}$ & $37.5 \pm 0.57 \mathrm{a}$ & $41.9 \pm 1.22 b$ \\
\hline TPRO & $32.9 \pm 1.35$ & $33.3 \pm 0.61$ & $35.1 \pm 0.35$ & $33.3 \pm 2.79$ & $29.7 \pm 1.69 \mathrm{a}$ & $32.2 \pm 1.87 \mathrm{ab}$ & $32.1 \pm 0.50 \mathrm{ab}$ & $34.8 \pm 0.33 b$ \\
\hline TFLAV & $38.5 \pm 1.34$ & $36.4 \pm 0.84$ & $38.4 \pm 0.14$ & $38.2 \pm 2.89$ & $29.7 \pm 0.38 a$ & $30.6 \pm 1.46 \mathrm{a}$ & $33.2 \pm 1.72 b$ & $32.8 \pm 0.71 b$ \\
\hline TSTILB & $5.13 \pm 0.09$ & $4.86 \pm 0.16$ & $5.50 \pm 0.15$ & $5.38 \pm 0.33$ & $5.07 \pm 0.05$ & $5.09 \pm 0.08$ & $5.73 \pm 0.30$ & $5.54 \pm 0.20$ \\
\hline TALC & $16.6 \pm 0.02$ & $16.4 \pm 0.13$ & $17.1 \pm 0.15$ & $17.7 \pm 0.88$ & $22.2 \pm 1.24$ & $21.9 \pm 0.76$ & $22.5 \pm 1.11$ & $23.2 \pm 0.01$ \\
\hline $3 \mathrm{MB}$ & CONT & SIDY & $\mathrm{CW}$ & YA & CONT & SIDY & $\mathrm{CW}$ & YA \\
\hline CI & $11.1 \pm 0.34 \mathrm{ab}$ & $9.90 \pm 0.41 \mathrm{a}$ & $9.83 \pm 1.08 \mathrm{a}$ & $13.1 \pm 0.95 b$ & $6.45 \pm 0.34 a$ & $6.06 \pm 0.02 a$ & $11.2 \pm 0.51 \mathrm{c}$ & $8.73 \pm 0.66 b$ \\
\hline $\mathbf{L}$ & $51.4 \pm 0.73 \mathrm{ab}$ & $54.7 \pm 1.47 \mathrm{~b}$ & $55.6 \pm 4.30 \mathrm{~b}$ & $46.0 \pm 2.58 \mathrm{a}$ & $69.1 \pm 1.02 c$ & $71.0 \pm 0.24 c$ & $51.7 \pm 1.22 \mathrm{a}$ & $60.4 \pm 2.84 b$ \\
\hline $\mathbf{a}$ & $43.1 \pm 0.84 \mathrm{ab}$ & $40.3 \pm 1.06 \mathrm{ab}$ & $39.2 \pm 4.19 \mathrm{a}$ & $45.7 \pm 1.31 b$ & $34.4 \pm 1.48 a$ & $32.2 \pm 0.35 a$ & $46.4 \pm 0.56 c$ & $41.8 \pm 0.20 b$ \\
\hline $\mathbf{b}$ & $13.7 \pm 0.31 b$ & $12.6 \pm 0.04 \mathrm{a}$ & $13.0 \pm 0.18 \mathrm{ab}$ & $13.2 \pm 0.57 \mathrm{ab}$ & $11.8 \pm 0.22$ & $13.0 \pm 1.51$ & $14.3 \pm 0.54$ & $13.8 \pm 3.21$ \\
\hline HBA & $46.1 \pm 1.56 \mathrm{~b}$ & $45.7 \pm 0.57 b$ & $45.7 \pm 0.02 b$ & $41.2 \pm 2.45 a$ & $40.6 \pm 0.83$ & $43.0 \pm 2.87$ & $39.3 \pm 2.40$ & $42.8 \pm 0.04$ \\
\hline HCA & $14.0 \pm 0.74 b$ & $13.7 \pm 0.05 b$ & $13.2 \pm 0.62 \mathrm{ab}$ & $11.9 \pm 0.41 \mathrm{a}$ & $14.1 \pm 0.47 \mathrm{a}$ & $15.7 \pm 0.27 b$ & $14.4 \pm 0.26 \mathrm{a}$ & $15.2 \pm 0.31 \mathrm{ab}$ \\
\hline HCATE & $2.49 \pm 0.07$ & $2.29 \pm 0.13$ & $2.25 \pm 0.01$ & $2.16 \pm 0.08$ & $3.95 \pm 0.20 \mathrm{ab}$ & $4.46 \pm 0.13 b$ & $2.83 \pm 0.02 \mathrm{a}$ & $4.48 \pm 0.16 b$ \\
\hline TFL & $40.1 \pm 2.10 b$ & $40.8 \pm 2.00 \mathrm{~b}$ & $40.2 \pm 1.83 b$ & $30.5 \pm 1.73 \mathrm{a}$ & $30.8 \pm 1.81 \mathrm{ab}$ & $39.4 \pm 2.03 c$ & $28.0 \pm 0.96 \mathrm{a}$ & $35.6 \pm 2.08 \mathrm{~b}$ \\
\hline TPRO & $31.2 \pm 1.85 b$ & $31.2 \pm 1.58 b$ & $28.2 \pm 1.05 a b$ & $26.0 \pm 0.69 \mathrm{a}$ & $26.4 \pm 1.62 \mathrm{~b}$ & $29.6 \pm 0.35 c$ & $20.1 \pm 0.12 \mathrm{a}$ & $26.8 \pm 1.23 b$ \\
\hline TFLAV & $34.7 \pm 1.07 \mathrm{~d}$ & $32.7 \pm 0.57 c$ & $30.9 \pm 0.21 b$ & $26.5 \pm 0.20 a$ & $23.5 \pm 0.22$ & $26.6 \pm 1.23$ & $23.6 \pm 1.74$ & $25.4 \pm 0.48$ \\
\hline TSTILB & $3.64 \pm 0.14$ & $3.66 \pm 0.01$ & $3.56 \pm 0.22$ & $3.21 \pm 0.07$ & $5.21 \pm 0.08$ & $5.57 \pm 0.29$ & $5.05 \pm 0.34$ & $5.50 \pm 0.09$ \\
\hline TALC & $16.4 \pm 0.64 b$ & $16.6 \pm 0.20 b$ & $16.8 \pm 0.19 \mathrm{~b}$ & $14.5 \pm 0.01 \mathrm{~b}$ & $19.7 \pm 0.10 \mathrm{a}$ & $21.5 \pm 1.55 \mathrm{ab}$ & $20.3 \pm 1.03 a$ & $22.6 \pm 0.37 b$ \\
\hline
\end{tabular}

Values in the same row with different letters indicate statistically significant differences $(p<0.05)$. 2MT (two months of treatment). 3MB (three months of bottle storage). HBA

hydroxybenzoic acids; HCA: hydroxycinnam 


\subsection{Effect of YDs Application on the Colour of Wines}

Table 1 shows the colour intensity (CI) and CIELab parameters of the different wines. The Uvaferm HPS $^{\circledR}$ fermented wines treated with CW and YA presented higher colour intensity $(\mathrm{CI})$ than the control wines and those treated with SIDY.

This result is well correlated with the different CIELab parameters evaluated, showing that CW- and YA-treated wines presented lower values of L (lightness) and b (blue-yellow chromatic coordinates), and higher values of a (red-green chromatic coordinates) than the control wines. Lower values of $b^{*}$ and higher values of $a^{*}$ indicated higher red-blue tonalities of wines, increasing their CI [35]. For these reasons, the use of these YDs could help to obtain wine with more stable colour pigments. However, these results were not obtained in the same treatments carried out with the Lalvin EC1118 ${ }^{\circledR}$ fermented wine.

Results obtained in the last few years in similar studies have shown the great ambiguity of the impact of polysaccharides on colour stability. Some authors reported that wine polysaccharides such as mannoproteins could improve the colour of wines [16,17,33]; others reported that these compounds could lead to a loss of colour $[4,9,10,18,20,32]$. Even within the same study, where six different YDs were tested, different results were reported: in some cases an increase in CI was observed and in others it was not [2].

\subsection{Effect of YDs on the Volatile Compounds}

A total of 38 volatile compounds were identified in red wines (Tables 2 and 3). The content of the different chemical groups of volatile compounds was different after the AMLF period as a function of the yeast strain used (but these results were not evaluated statistically).In general, the content of different volatile groups was higher in wines fermented with Uvaferm HPS ${ }^{\circledR}$ than in those fermented with Lalvin EC1118 ${ }^{\circledR}$, with the exception of terpenes. With respect to the esters group, the trend between every ester group (ethyl, methyl, acetate, and isoamyl esters) was very similar to the global trend. However, it is important to note that the concentration of isoamyl and acetate esters of wines fermented with Uvaferm HPS ${ }^{\circledR}$ was significantly higher than the wines fermented with Lalvin EC1118 ${ }^{\circledR}$ (Figures 3 and 4), mainly in the case of isoamyl and acetate esters (double and triple, respectively; see the Tables 2 and 3). The trend of the different volatile compound groups studied after $2 \mathrm{MT}$ and $3 \mathrm{MB}$ periods was not always the same in the two types of wines fermented and, depending on the treatment applied, the content of these compounds increased or decreased (Figures 3 and 4).

In the case of wines fermented with Lalvin $\mathrm{EC} 1118^{\circledR}{ }^{\circledR}$, no significant differences were found in the content of total ethyl esters after the 2MT period, but the wines treated with SIDY and CW had a significantly higher content of acetate esters than the control wines, mainly due to their high content of isoamyl acetate. In addition, the wines treated with CW had a higher content of total fatty acids than the control wines. However, it seems that the period of bottle storage (3MB) produced significant changes, mainly in the wines treated with CW and YA, revealing significantly higher contents of ethyl, methyl and isoamyl esters than in the control wines (see Table 2). Also, treatment with CW increased the concentration of the acetate esters, producing a significant increase of the total esters content of these treated wines at this point of bottle storage. The esters concentration in wines is usually above their perception threshold, which is one of the reasons why they are major contributors to the global aroma of a wine in sensory evaluations [36]. Esters such as ethyl butanoate, hexanoate, and octanoate, which increase after bottle storage with the addition of YDs (see Table 2), have been related to the red-berry aroma in red wine [37]. The same increase observed in esters after the $3 \mathrm{MB}$ period was also observed in the total alcohols and acids. Also, the total amount of terpenes increased with all the treatments. 
Table 2. Volatile compound composition in red wines fermented with Lalvin EC1118 ${ }^{\circledR}$ (average \pm standard deviation) expressed in $\mu \mathrm{g} / \mathrm{L}$.

\begin{tabular}{|c|c|c|c|c|c|c|c|c|c|c|c|}
\hline & \multirow[t]{2}{*}{ LRI } & \multirow[t]{2}{*}{ ID } & \multirow{2}{*}{$\begin{array}{c}\text { AMLF } \\
\mathrm{C}\end{array}$} & \multicolumn{4}{|c|}{ 2MT } & \multicolumn{4}{|c|}{ 3MB } \\
\hline & & & & C & SIDY1 & $\mathrm{CW}$ & YA & C & SIDY1 & $\mathrm{CW}$ & YA \\
\hline \multicolumn{12}{|l|}{ Ethyl esters } \\
\hline Ethyl butanoate & 1076 & A & $178 \pm 13$ & $144 \pm 9$ & $171 \pm 10$ & $159 \pm 22$ & $156 \pm 7$ & $138 \pm 19 a$ & $158 \pm 90 \mathrm{a}$ & $204 \pm 2 b$ & $201 \pm 2 b$ \\
\hline Ethyl hexanoate & 1246 & A & $291 \pm 43$ & $214 \pm 4$ & $254 \pm 27$ & $230 \pm 64$ & $199 \pm 3$ & $198 \pm 34 a$ & $225 \pm 32 a$ & $359 b \pm 3$ & $375 \pm 24 \mathrm{~b}$ \\
\hline Ethyl heptanoate & 1334 & B & $10.9 \pm 0.90$ & $10.5 \pm 1.20$ & $12.7 \pm 0.80$ & $10.3 \pm 1.20$ & $11.3 \pm 0.20$ & $7.87 \pm 0.62 a$ & $8.92 a \pm 0.69$ & $10.7 \mathrm{~b} \pm 0.30$ & $11.3 b \pm 0.60$ \\
\hline Ethyl lactate & 1413 & A & $19.9 \pm 1.60$ & $29.9 \pm 8.40$ & $38.2 \pm 7.30$ & $42.1 \pm 1.20$ & $38.9 \pm 4.2$ & $19.2 \pm 1.70 \mathrm{a}$ & $20.8 \pm 3.50 \mathrm{ab}$ & $31.0 \pm 6.30 \mathrm{~b}$ & $28.4 \pm 1.50 \mathrm{~b}$ \\
\hline Ethyl octanoate & 1460 & A & $888 \pm 83$ & $739 \pm 12$ & $782 \pm 59$ & $728 \pm 95$ & $794 \pm 69$ & $629 \pm 111 a$ & $720 \pm 7 \mathrm{a}$ & $998 \pm 15 b$ & $1096 \pm 152 b$ \\
\hline Ethyl nonanoate & 1558 & A & $45.6 \pm 2.7$ & $27.0 \pm 3.50$ & $36.0 \pm 0.10$ & $32.5 \pm 12.70$ & $28.1 \pm 6.50$ & $17.9 \pm 0.60 \mathrm{a}$ & $21.7 \pm 0.30 \mathrm{ab}$ & $22.9 \pm 1.10 \mathrm{~b}$ & $24.1 \pm 3.60 \mathrm{~b}$ \\
\hline Ethyl succinate & 1701 & A & $92 \pm 5$ & $108 \pm 2$ & $111 \pm 9$ & $113 \pm 23$ & $114 \pm 11$ & $134 \pm 9 a$ & $142 \pm 2 a$ & $176 \pm 10 \mathrm{~b}$ & $195 \pm 7 \mathrm{~b}$ \\
\hline Ethyl decanoate & 1715 & A & $127 \pm 7$ & $51.0 \pm 3.80 \mathrm{a}$ & $52.6 \pm 5.30 \mathrm{ab}$ & $47.7 \pm 2.0 \mathrm{a}$ & $60.7 \pm 5.60 \mathrm{~b}$ & $34.5 \pm 7.80 \mathrm{a}$ & $47.1 \pm 0.30 \mathrm{ab}$ & $61.8 \pm 4.10 \mathrm{~b}$ & $70.9 \pm 21 \mathrm{~b}$ \\
\hline Ethyl isovalerate & 1806 & A & $4.09 \pm 0.08$ & $2.64 \pm 1.36$ & $2.82 \pm 1.90$ & $5.55 \pm 2.19$ & $6.27 \pm 2.94$ & $1.50 \pm 0.210$ & $3.36 \pm 1.02$ & $2.09 \pm 0.34$ & $3.18 \pm 3.02$ \\
\hline Ethyl undecanoate & 1824 & A & $1.20 \pm 0.10$ & $0.876 \pm 0.06$ & $1.07 \pm 0.09$ & $1.07 \pm 0.27$ & $1.07 \pm 0.08$ & $0.680 \pm 0.014 a$ & $0.745 \pm 0.05 a$ & $0.810 \pm 0.03 a$ & $1.01 \pm 0.07 \mathrm{~b}$ \\
\hline Ethyl dodecanoate & 1869 & B & $116 \pm 8$ & $31.5 \pm 1.0$ & $38.3 \pm 3.30$ & $32.3 \pm 2.50$ & $39.5 \pm 12.40$ & $14.5 \pm 2.10 \mathrm{ab}$ & $10.5 \pm 2.10 \mathrm{a}$ & $20.2 \pm 0.20 \mathrm{~b}$ & $20.4 \pm 5.60 \mathrm{~b}$ \\
\hline Ethyl tetradecanoate & 2068 & B & $16.0 \pm 2.10$ & $9.90 \pm 0.53$ & $11.6 \pm 1.10$ & $11.1 \pm 0.20$ & $11.1 \pm 1.30$ & $8.26 \pm 0.01 \mathrm{a}$ & $7.02 \pm 1.62 a$ & $12.1 \pm 0.60 \mathrm{~b}$ & $9.37 \pm 0.87 \mathrm{a}$ \\
\hline $\begin{array}{l}\text { Ethyl hexadecanoate } \\
\text { Methyl esters }\end{array}$ & $<2100$ & B & $21.6 \pm 1.80$ & $14.5 \pm 0.80$ & $15.8 \pm 0.10$ & $17.2 \pm 2.0$ & $14.1 \pm 1.10$ & $11.5 \pm 0.30 \mathrm{a}$ & $11.1 \pm 2.0 \mathrm{a}$ & $14.7 \pm 3.80 \mathrm{~b}$ & $16.6 \pm 0.70 \mathrm{~b}$ \\
\hline Methyl hexanoate & 1183 & A & $1.63 \pm 0.23$ & $0.99 \pm 0.28$ & $1.45 \pm 0.25$ & $1.45 \pm 0.16$ & $1.36 \pm 0.02$ & $0.81 \mathrm{a} \pm 0.36$ & $1.27 \mathrm{ab} \pm 0.07$ & $2.00 \mathrm{~b} \pm 0.25$ & $2.09 b \pm 0.50$ \\
\hline Methyl octanoate & 1420 & A & $6.80 \pm 0.66$ & $5.10 \pm 0.12$ & $5.65 \pm 0.32$ & $5.26 \pm 0.86$ & $6.40 \pm 0.59$ & $4.69 \pm 0.50 \mathrm{a}$ & $4.55 \pm 0.69 \mathrm{a}$ & $6.52 \pm 0.06 \mathrm{ab}$ & $7.82 \pm 1.52 b$ \\
\hline $\begin{array}{l}\text { Methyl decanoate } \\
\text { Acetate esters }\end{array}$ & & nd \\
\hline Isoamyl acetate & 1163 & A & $2117 \pm 253$ & $584 \pm 30 a$ & $1472 \pm 306 \mathrm{~b}$ & $1572 \pm 110 \mathrm{~b}$ & $884 \pm 170 a$ & $706 \pm 291 a$ & $939 \pm 61 \mathrm{a}$ & $1872 \pm 90 \mathrm{~b}$ & $1084 \pm 163 a$ \\
\hline Hexyl acetate & 1306 & A & $2.39 \pm 0.07$ & $1.73 \pm 0.10$ & $1.92 \pm 0.02$ & $1.78 \pm 0.00$ & $1.92 \pm 0.16$ & $2.01 \pm 0.40$ & $1.68 \pm 0.26$ & $2.30 \pm 0.05$ & $2.82 \pm 0.76$ \\
\hline $\begin{array}{l}\text { 2-phenylethyl acetate } \\
\text { Isoamyl esters }\end{array}$ & \multicolumn{9}{|c|}{ Isoamyl esters } & $91.4 \pm 16.70 \mathrm{a}$ & $174 \pm 15 b$ \\
\hline Isopentyl hexanoate & 1478 & A & $0.740 \pm 0.01$ & $0.581 \pm 0.03$ & $0.605 \pm 0.09$ & $0.471 \pm 0.195$ & $0.597 \pm 0.05$ & $0.423 \pm 0.185 a$ & $0.526 \pm 0.09 a$ & $0.843 \pm 0.045 b$ & $0.898 \pm 0.06 \mathrm{~b}$ \\
\hline Isoamyl octanoate & 1748 & A & $1172 \pm 110$ & $1152 \pm 1$ & $1252 \pm 171$ & $1055 \pm 168$ & $955 \pm 64$ & $662 a \pm 147$ & $958 \mathrm{~b} \pm 17$ & $1238 c \pm 45$ & $1205 \mathrm{c} \pm 97$ \\
\hline $\begin{array}{l}\text { Isoamyl decanoate } \\
\text { Alcohols }\end{array}$ & 1909 & A & $138 \pm 10$ & $44.5 \pm 2.0$ & $48.2 \pm 3.80$ & $40.0 \pm 2.70$ & $51.8 \pm 3.30$ & $16.4 \pm 5.10$ & $12.7 \pm 0.80$ & $20.9 \pm 2.0$ & $28.2 \pm 8.2$ \\
\hline Isobutanol & 1108 & A & $70577 \pm 4434$ & $60577 \pm 1383$ & $65910 \pm 4970$ & $63910 \pm 11482$ & $62910 \pm 2805$ & $64243 \pm 6881 \mathrm{a}$ & $68910 \pm 1391 a$ & $89243 \pm 6641 \mathrm{~b}$ & $90243 \pm 2579 b$ \\
\hline 3-Methyl-1-butanol & 1197 & A & $107593 \pm 6486$ & $98038 \pm 396$ & $106260 \pm 5796$ & $107593 \pm 3115$ & $99704 \pm 2800$ & $97816 \pm 7230 \mathrm{a}$ & $104593 \pm 4162 a$ & $134927 \pm 3090 \mathrm{~b}$ & $133816 \pm 3935 b$ \\
\hline Hexanol & 1391 & A & $2377 \pm 177$ & $2227 \pm 10$ & $2477 \pm 193$ & $2377 \pm 134$ & $2327 \pm 24$ & $2402 \pm 154 a$ & $2577 \pm 170 a$ & $3452 \pm 247 \mathrm{~b}$ & $3502 \pm 76 \mathrm{~b}$ \\
\hline Heptanol & 1478 & B & $29.4 \pm 1.30$ & $30.4 \pm 1.0$ & $32.9 \pm 1.90$ & $31.9 \pm 2.60$ & $31.4 \pm 1.50$ & $31.9 \pm 0.40 \mathrm{a}$ & $34.4 \pm 2.0 \mathrm{a}$ & $43.4 \pm 3.10 \mathrm{~b}$ & $43.9 \pm 1.90 \mathrm{~b}$ \\
\hline Octanol & 1578 & A & $7.24 \pm 0.43$ & $8.61 \pm 0.01$ & $9.72 \pm 1.20$ & $9.26 \pm 1.30$ & $9.17 \pm 0.45$ & $9.44 \pm 0.15 a$ & $10.3 \pm 0.40 \mathrm{a}$ & $14.3 \pm 0.60 \mathrm{~b}$ & $14.5 \pm 0.10 \mathrm{~b}$ \\
\hline Decanol & 1773 & A & $3.09 \pm 0.29$ & $3.32 \pm 0.02$ & $3.56 \pm 0.08$ & $3.50 \pm 0.29$ & $3.44 \pm 0.06$ & $3.50 \pm 0.00$ & $3.74 \pm 0.13$ & $4.68 \pm 0.02$ & $4.74 \pm 0.13$ \\
\hline Benzyl alcohol & 1978 & B & $360 \pm 18$ & $618 \pm 16$ & $675 \pm 79$ & $650 \pm 164$ & $643 \pm 82$ & $658 \pm 114$ & $705 \pm 171$ & $910 \pm 334$ & $925 \pm 102$ \\
\hline 2-Phenylethanol & 2020 & A & $15689 \pm 731$ & $14329 \pm 173 a$ & $15829 \pm 2002 b$ & $15209 \pm 3789 a b$ & $14989 \pm 1973 a$ & $15349 \pm 2347 \mathrm{a}$ & $16609 \pm 176 a$ & $22109 \pm 421 b$ & $22509 \pm 2191 b$ \\
\hline \multicolumn{12}{|l|}{ Volatile fatty acids } \\
\hline Hexanoic acid & 1894 & A & $1170 \pm 20$ & $1200 \pm 15$ & $1268 \pm 135.60$ & $1385 \pm 250$ & $1288 \pm 118$ & $1168 \pm 125 a$ & $1290 \pm 28 \mathrm{ab}$ & $1568 \pm 88 \mathrm{bc}$ & $1660 \pm 151 \mathrm{c}$ \\
\hline Octanoic acid & $<2100$ & A & $209 \pm 19$ & $113 \pm 45$ & $203 \pm 98.90$ & $304 \pm 125$ & $284 \pm 80$ & $124 \pm 109 a$ & $243 \pm 37 \mathrm{ab}$ & $364 \pm 22 b$ & $534 \pm 26 c$ \\
\hline Decanoic acid & $<2100$ & A & $102 \pm 1$ & $42.0 \pm 0.5$ & $42.8 \pm 3.0$ & $46.8 \pm 4.80$ & $66.8 \pm 15.50$ & $41.6 \pm 3.90 \mathrm{a}$ & $45.6 \pm 3.60 \mathrm{ab}$ & $52.4 \pm 6.90 \mathrm{ab}$ & $75.6 \pm 22.4 \mathrm{~b}$ \\
\hline Dodecanoic acid & $<2100$ & B & $14.04 \pm 0.84$ & $14.84 \pm 1.46$ & $16.1 \pm 0.10$ & $16.8 \pm 1.0$ & $14.84 \pm 0.48$ & $15.24 \pm 0.12$ & $14.84 \pm 0.62$ & $16.4 \pm 1.40$ & $16.04 \pm 0.31$ \\
\hline \multicolumn{12}{|l|}{ Terpenes } \\
\hline Citronellol & 1785 & A & $3.44 \pm 0.09$ & $3.60 \pm 0.01$ & $3.76 \pm 0.50$ & $3.76 \pm 0.44$ & $3.60 \pm 0.10$ & $3.76 \pm 0.12 \mathrm{a}$ & $3.92 \pm 0.10 \mathrm{a}$ & $4.87 \pm 0.00 \mathrm{~b}$ & $5.03 \pm 0.13 b$ \\
\hline Nerol & 1887 & A & $16.6 \pm 1.0$ & $24.3 \pm 1.10$ & $25.7 \pm 1.40$ & $25.2 \pm 3.10$ & $24.8 \pm 2.50$ & $25.2 \pm 0.70 \mathrm{a}$ & $26.6 \pm 0.70 \mathrm{a}$ & $32.5 \pm 2.40 \mathrm{~b}$ & $33.0 \pm 0.30 \mathrm{~b}$ \\
\hline trans-nerolidol & 2056 & A & $2.61 \pm 0.09$ & $3.66 \pm 0.18$ & $3.95 \pm 0.14$ & $3.86 \pm 0.16$ & $3.86 \pm 0.08$ & $3.86 \pm 0.07 \mathrm{a}$ & $4.05 \pm 0.08 a$ & $4.91 \pm 0.06 \mathrm{~b}$ & $5.01 \pm 0.15 b$ \\
\hline
\end{tabular}

Values with different letter in the same row, and within of each point of analysis, indicate statistically significant differences $(p<0.05)$. Values without letter indicate no statistically significant differences. AAF: After alcoholic fermentation, 2MT: two months of treatment, 3MB: three months of bottle storage. LRI: Linear Retention Index. ID: reliability of identification:

A, mass spectrum and LRI agreed with standards; B, mass spectrum agreed with mass spectral data base and/or LRI agreed with the literature data. 
Table 3. Volatile compounds composition in the red wines fermented with Uvaferm HPS $^{\circledR}$ (average \pm standard deviation) expressed in $\mu \mathrm{g} / \mathrm{L}$.

\begin{tabular}{|c|c|c|c|c|c|c|c|c|c|c|c|}
\hline & \multirow[t]{2}{*}{ LRI } & \multirow[t]{2}{*}{ ID } & \multirow{2}{*}{$\begin{array}{c}\text { AMLF } \\
\text { C }\end{array}$} & \multicolumn{4}{|c|}{ 2MT } & \multicolumn{4}{|c|}{$3 \mathrm{BS}$} \\
\hline & & & & C & SIDY1 & $\mathrm{CW}$ & YA & $\mathrm{C}$ & SIDY1 & $\mathrm{CW}$ & YA \\
\hline \multicolumn{12}{|l|}{ Ethyl esters } \\
\hline Ethyl butanoate & 1076 & A & $234 \pm 10$ & $203 \pm 8 \mathrm{a}$ & $214 \pm 7 \mathrm{ab}$ & $203 \pm 12 a$ & $232 \pm 2 b$ & $192 \pm 16$ & $213 \pm 1$ & $207 \pm 5$ & $199 \pm 1$ \\
\hline Ethyl hexanoate & 1246 & A & $396 \pm 29$ & $310 \pm 32 \mathrm{ab}$ & $325 \pm 7 \mathrm{ab}$ & $292 \pm 49 a$ & $392 \pm 17 \mathrm{~b}$ & $304 \pm 28 a$ & $346 \pm 13 b$ & $327 \pm 19 a b$ & $303 \pm 13 a$ \\
\hline Ethyl heptanoate & 1334 & B & $9.57 \pm 0.21$ & $9.24 \pm 1.89$ & $8.33 \pm 1.08$ & $9.37 \pm 1.78$ & $9.96 \pm 0.42$ & $7.41 \pm 0.45$ & $7.80 \pm 0.43$ & $8.26 \pm 0.32$ & $\begin{array}{l}3001 \pm 0.04 \\
7.61 \pm 0\end{array}$ \\
\hline Ethyl lactate & 1413 & A & $3.14 \pm 1.17$ & $2.86 \pm 0.21$ & $2.82 \pm 1.04$ & $1.75 \pm 0.73$ & $3.12 \pm 0.55$ & $10.5 \pm 2.8 \mathrm{c}$ & $6.64 \pm 2.73 \mathrm{ab}$ & $14.5 \pm 1.7 \mathrm{c}$ & $3.40 \pm 0.04 \mathrm{a}$ \\
\hline Ethyl octanoate & 1460 & A & $1143 \pm 47$ & $928 \pm 117 \mathrm{a}$ & $1049 \pm 17 \mathrm{ab}$ & $826 \pm 107 a$ & $1229 \pm 120 \mathrm{~b}$ & $786 \pm 61 a$ & $1069 \pm 96 \mathrm{~b}$ & $853 \pm 70 \mathrm{ab}$ & $924 \pm 79 \mathrm{ab}$ \\
\hline Ethyl nonanoate & 1558 & A & $56.7 \pm 0.9$ & $31.7 \pm 5.3$ & $26.6 \pm 4.5$ & $23.8 \pm 0.7$ & $28.8 \pm 9.8$ & $14.3 \pm 2.0$ & $15.4 \pm 2.0$ & $17.7 \pm 1.7$ & $17.9 \pm 1.0$ \\
\hline Ethyl succinate & 1701 & A & $333 \pm 6$ & $172 \pm 5 a$ & $345 \pm 1 \mathrm{~b}$ & $132 \pm 11 a$ & $297 \pm 2 b$ & $159 \pm 7 \mathrm{a}$ & $278 \pm 7 \mathrm{~b}$ & $170 \pm 6 a$ & $267 \pm 4 \mathrm{~b}$ \\
\hline Ethyl decanoate & 1715 & A & $22.1 \pm 2.4$ & $36.4 \pm 2.2 \mathrm{~b}$ & $29.7 \pm 3.8 \mathrm{a}$ & $36.1 \pm 2.4 \mathrm{~b}$ & $38.1 \pm 29.3 \mathrm{~b}$ & $54.8 \pm 3.8 \mathrm{ab}$ & $54.0 \pm 14.2 \mathrm{ab}$ & $59.7 \pm 0.6 \mathrm{~b}$ & $48.9 \pm 7.7 \mathrm{a}$ \\
\hline Ethyl undecanoate & 1824 & A & $0.680 \pm 0.030$ & $0.549 \pm 0.016 \mathrm{~b}$ & $0.614 \pm 0.042 \mathrm{c}$ & $0.418 \pm 0.021 \mathrm{a}$ & $0.484 \pm 0.133 a$ & $2.18 \pm 0.04$ & $3.16 \pm 0.05$ & $2.44 \pm 0.01$ & $2.05 \pm 0.07$ \\
\hline Ethyl dodecanoate & 1869 & B & $14.3 \pm 1.0$ & $6.95 \pm 0.17 a$ & $20.5 \pm 0.4 c$ & $5.25 \pm 0.22 \mathrm{a}$ & $12.9 \pm 2.6 \mathrm{~b}$ & $22.6 \pm 1.4 a$ & $34.1 \pm 1.5 \mathrm{~b}$ & $23.9 \pm 0.9 \mathrm{a}$ & $24.9 \pm 1.8 \mathrm{a}$ \\
\hline Ethyl tetradecanoate & 2068 & B & $16.0 \pm 1.1$ & $7.54 \pm 0.01 \mathrm{a}$ & $9.83 \pm 0.27 \mathrm{bc}$ & $9.05 \pm 0.52 \mathrm{ab}$ & $11.1 \pm 1.1 \mathrm{c}$ & $16.7 \pm 2.5 \mathrm{~b}$ & $14.8 \pm 1.5 \mathrm{~b}$ & $18.3 \pm 1.8 \mathrm{~b}$ & $7.35 \pm 0.52 a$ \\
\hline \multicolumn{12}{|l|}{ Methyl esters } \\
\hline Methyl hexanoate & 1183 & A & $1.63 \pm 0.27$ & $1.17 \pm 0.11 \mathrm{a}$ & $1.27 \pm 0.02 \mathrm{a}$ & $1.08 \pm 0.21 \mathrm{a}$ & $1.72 \mathrm{~b} \pm 0.00$ & $1.08 \pm 0.20$ & $1.17 \pm 0.47$ & $1.08 \pm 0.32$ & $0.807 \pm 0.167$ \\
\hline Methyl octanoate & 1420 & A & $6.64 \pm 0.29$ & $4.33 \pm 0.86 \mathrm{a}$ & $4.98 \pm 0.37 \mathrm{ab}$ & $4.09 \pm 0.83 a$ & $6.54 \pm 1.35 \mathrm{~b}$ & $3.64 \pm 0.51 \mathrm{a}$ & $5.42 \pm 0.41 \mathrm{~b}$ & $4.02 \pm 0.41 \mathrm{a}$ & $4.31 \pm 0.55 \mathrm{ab}$ \\
\hline \multicolumn{11}{|l|}{ Acetate esters } & nd \\
\hline Isoamyl acetate & 1163 & A & $5928 \pm 400$ & $4350 \pm 560 \mathrm{a}$ & $5328 \pm 123 b$ & $4261 \pm 121 \mathrm{a}$ & $5717 \pm 149 \mathrm{~b}$ & $3572 \pm 111 \mathrm{a}$ & $4695 \pm 412 b$ & $3350 \pm 292 a$ & $3750 \pm 189 a$ \\
\hline Hexyl acetate & 1306 & A & $8.22 \pm 0.43$ & $4.38 \pm 1.83 a$ & $21.6 \pm 0.4 \mathrm{~b}$ & $4.86 \pm 0.51 \mathrm{a}$ & $14.6 \pm 8.1 \mathrm{ab}$ & $3.63 \pm 0.01 \mathrm{a}$ & $17.3 \pm 2.0 \mathrm{~b}$ & $3.01 \pm 0.05 \mathrm{a}$ & $8.13 \pm 1.02 \mathrm{ab}$ \\
\hline $\begin{array}{l}\text { 2-Phenylethyl acetate } \\
\text { Isoamyl esters }\end{array}$ & 1851 & A & $1069 \pm 37$ & $285 \pm 21 a$ & $869 \pm 47 \mathrm{c}$ & $246 \pm 11 a$ & $604 \pm 56 \mathrm{~b}$ & $188 \pm 48 a$ & $309 \pm 8 \mathrm{~b}$ & $138 \pm 15 a$ & $201 \pm 36 a$ \\
\hline Isoamyl hexanoate & 1478 & A & $1.53 \pm 0.07$ & $1.31 \pm 0.25$ & $1.07 \pm 0.07$ & $1.14 \pm 0.22$ & $1.51 \pm 0.19$ & $0.922 \pm 0.104$ & $1.19 \pm 0.10$ & $1.10 \pm 0.16$ & $0.977 \pm 0.079$ \\
\hline Isoamyl octanoate & 1748 & A & $1898 \pm 79$ & $2008 \pm 21 \mathrm{~b}$ & $2422 \pm 29 \mathrm{c}$ & $1362 \pm 12 a$ & $2155 \pm 155 b$ & $1338 \pm 37 a$ & $1802 \pm 44 \mathrm{~b}$ & $1385 \pm 87 a$ & $1685 \pm 163 \mathrm{~b}$ \\
\hline $\begin{array}{l}\text { Isoamyl decanoate } \\
\text { Alcohols }\end{array}$ & 1909 & \multicolumn{6}{|c|}{ Alcohols } & & & & $74.5 \pm 5.7 \mathrm{~b}$ \\
\hline Isobutanol & 1108 & A & $64910 \pm 4893$ & $61577 \pm 1415$ & $60910 \pm 6598$ & $54243 \pm 2798$ & $62910 \pm 94$ & $59243 \pm 4028$ & $58910 \pm 4529$ & $63910 \pm 3131$ & $58910 \pm 3525$ \\
\hline 3-Methyl-1-butanol & 1197 & A & $136038 \pm 6490$ & $128260 \pm 953 a$ & $129371 \pm 5136 \mathrm{a}$ & $123816 \pm 6513 a$ & $142704 \pm 3344 \mathrm{~b}$ & $134927 \pm 1213$ & $140482 \pm 9572$ & $134927 \pm 3572$ & $128260 \pm 1694$ \\
\hline $\begin{array}{l}\text { 3-Mietryy-1-Dutanol } \\
\text { Hexanol }\end{array}$ & 1391 & $\begin{array}{l}\mathrm{A} \\
\mathrm{A}\end{array}$ & $\begin{array}{c}130030 \pm 6490 \\
2402 \pm 141\end{array}$ & $\begin{array}{c}12200 \pm 930 \mathrm{a} \\
2252 \pm 4 \mathrm{ab}\end{array}$ & $\begin{array}{l}2252 \pm 131 \mathrm{ab} \\
2252\end{array}$ & $\begin{array}{c}123015 \pm \pm 136 a \\
2152 \pm 136 a\end{array}$ & $\begin{array}{c}2502 \pm 102 \mathrm{~b} \\
2502 \pm 0\end{array}$ & $\begin{array}{c}13927 \pm 1213 \\
2402 \pm 46\end{array}$ & $\begin{array}{c}140482 \pm 90 / 2 \\
2477 \pm 218\end{array}$ & $\begin{array}{c}134927 \pm 30 / 2 \\
2377 \pm 91\end{array}$ & $\begin{aligned} 22825 & \pm 54 \\
2252 & \end{aligned}$ \\
\hline Heptanol & 1478 & $\mathrm{~B}$ & $20.9 \pm 0.5$ & $19.9 \pm 0.3$ & $18.9 \pm 1.1$ & $19.9 \pm 0.9$ & $21.4 \pm 0.8$ & $21.4 \pm 0.1$ & $20.9 \pm 1.7$ & $21.9 \pm 1.0$ & $19.9 \pm 0.1$ \\
\hline Octanol & 1578 & A & $9.17 \pm 0.85$ & $8.06 \pm 0.33 a$ & $8.71 \pm 0.65 a$ & $8.61 \pm 0.45 a$ & $10.2 \pm 0.3 \mathrm{~b}$ & $9.17 \pm 0.17$ & $9.62 \pm 0.56$ & $8.98 \pm 0.68$ & $8.89 \pm 0.03$ \\
\hline Decanol & 1773 & A & $3.38 \pm 0.22$ & $2.79 \pm 0.06$ & $2.97 \pm 0.11$ & $2.91 \pm 0.21$ & $3.21 \pm 0.22$ & $2.97 \pm 0.05$ & $3.03 \pm 0.14$ & $2.74 \pm 0.03$ & $2.74 \pm 0.03$ \\
\hline Benzyl alcohol & 1978 & B & $265 \pm 47$ & $220 \pm 4$ & $220 \pm 18$ & $215 \pm 21$ & $240 \pm 19$ & $260 \pm 17$ & $268 \pm 24$ & $283 \pm 40$ & $213 \pm 2$ \\
\hline 2-Phenylethanol & 2020 & A & $24709 \pm 4375$ & $20509 \pm 30$ & $21109 \pm 1625$ & $20909 \pm 2112$ & $23709 \pm 1537$ & $21309 \pm 452 \mathrm{ab}$ & $26109 \pm 2628 b$ & $23309 \pm 2543 \mathrm{ab}$ & $20509 \pm 38 \mathrm{a}$ \\
\hline \multicolumn{12}{|l|}{ Volatile fatty acids } \\
\hline Hexanoic acid & 1894 & A & $1885 \pm 262$ & $1695 \pm 16$ & $1785 \pm 132$ & $1707 \pm 179$ & $1932 \pm 120$ & $1810 \pm 7 \mathrm{ab}$ & $2070 \pm 128 \mathrm{~b}$ & $1852 \pm 158 \mathrm{ab}$ & $1760 \pm 23 a$ \\
\hline Octanoic acid & $<2100$ & A & $1074 \pm 246$ & $584 \pm 46 a$ & $1054 \pm 185 \mathrm{~b}$ & $634 \pm 116 a$ & $1124 \pm 15 b$ & $754 \pm 58 a$ & $1354 \pm 96 \mathrm{~b}$ & $724 \pm 87 a$ & $864 \pm 49 a$ \\
\hline Decanoic acid & $<2100$ & $\mathrm{~A}$ & $166 \pm 12$ & $56.8 \pm 2.0 \mathrm{a}$ & $138 \pm 5 b$ & $60.0 \pm 5.8 \mathrm{a}$ & $135 \pm 10 \mathrm{~b}$ & $69.2 \pm 0.6 \mathrm{a}$ & $150 \pm 11 \mathrm{c}$ & $74.0 \pm 3.7 \mathrm{a}$ & $112 \pm 9 \mathrm{~b}$ \\
\hline $\begin{array}{l}\text { Dodecanoic acid } \\
\text { Terpenes }\end{array}$ & $<2100$ & B & $18.1 \pm 0.4$ & $16.8 \pm 0.3$ & $16.4 \pm 0.3$ & $15.6 \pm 0.8$ & $16.4 \pm 0.3$ & $14.8^{\mathrm{b}} \pm 0.2$ & $18.1^{\mathrm{d}} \pm 0.7$ & $16.4^{\mathrm{c}} \pm 0.4$ & $12.4^{\mathrm{a}} \pm 0.4$ \\
\hline Citronellol & 1785 & A & $4.56 \pm 0.24$ & $4.24 \pm 0.05$ & $4.40 \pm 0.46$ & $4.40 \pm 0.32$ & $4.87 \pm 0.22$ & $4.24 \pm 0.24$ & $4.56 \pm 0.34$ & $4.24 \pm 0.12$ & $4.08 \pm 0.29$ \\
\hline Nerol & 1887 & A & $13.9 \pm 1.5$ & $12.5 \pm 0.3$ & $11.6 \pm 0.5$ & $13.4 \pm 1.4$ & $13.9 \pm 0.5$ & $12.0 \pm 0.7$ & $12.0 \pm 0.6$ & $11.6 \pm 0.1$ & $11.6 \pm 0.2$ \\
\hline trans-Nerolidol & 2056 & A & $2.51 \pm 0.08$ & $2.80 \pm 0.24 \mathrm{ab}$ & $2.32 \pm 0.08 \mathrm{a}$ & $3.18 \pm 0.03 \mathrm{~b}$ & $2.22 \pm 0.33 a$ & $3.09 \pm 0.49 \mathrm{a}$ & $2.61 \pm 0.23 \mathrm{a}$ & $4.63 \pm 0.01 \mathrm{~b}$ & $2.41 \pm 0.18 \mathrm{a}$ \\
\hline
\end{tabular}

Values with different letter in the same row, and within of each point of analysis, indicate statistically significant differences $(p<0.05)$. Values without letter indicate no statistically significant differences. AMLF: After malolactic fermentation, 2MT: two months of treatment, 3MB: three months of bottle storage. LRI: Linear Retention Index. ID: reliability of identification: A, mass spectrum and LRI agreed with standards; B, mass spectrum agreed with mass spectral data base and/or LRI agreed with the literature data. 


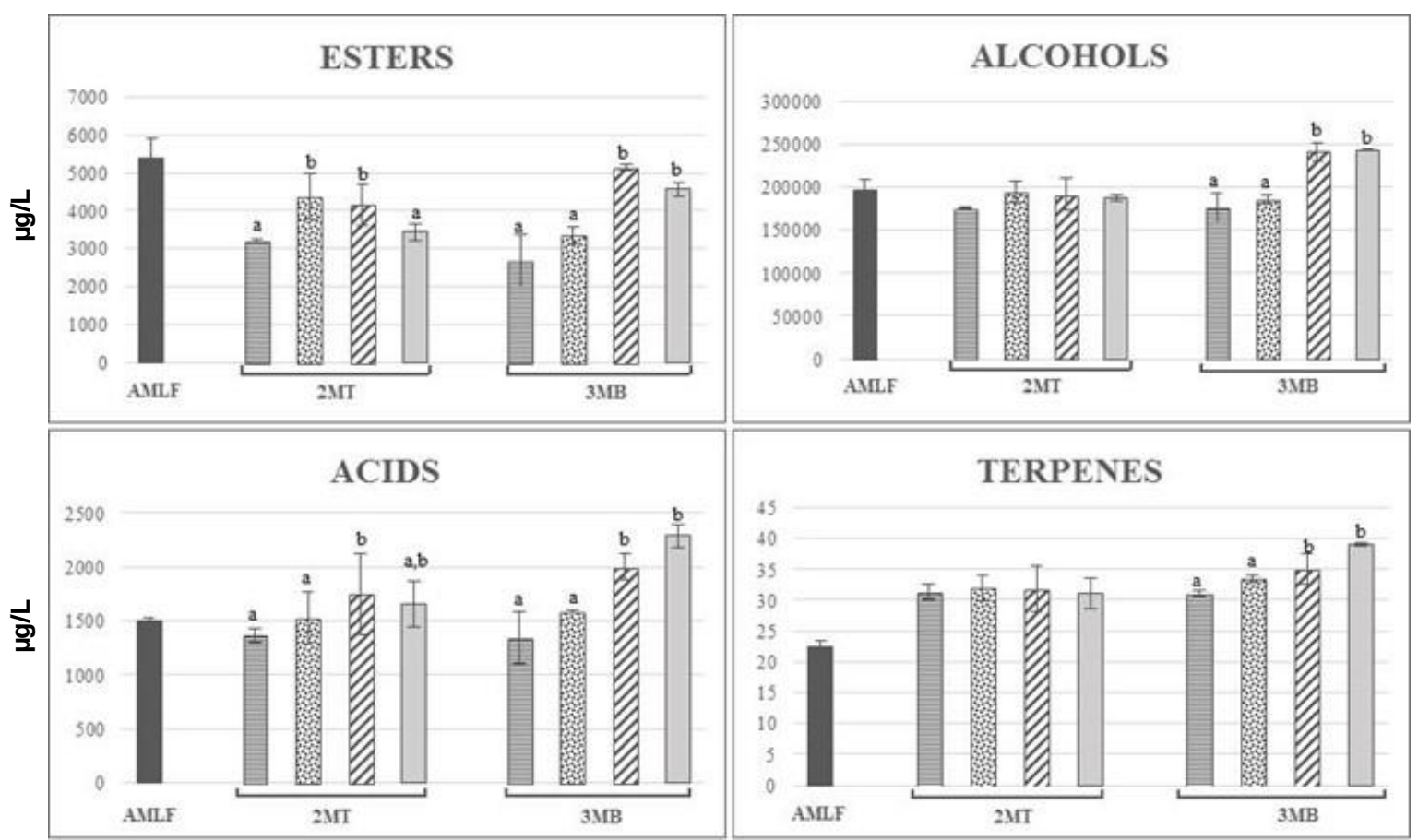

Figure 3. Evolution of the volatile compounds during the treatment and bottle storage in Carménère wines fermented with Lalvin EC1118 ${ }^{\circledR}$. Results expressed in $\mu \mathrm{g} / \mathrm{L}$. Control after malolactic fermentation; 目 Control; 5 SIDY; $\mathbf{D}$ CW; $\square$ YA. MT: Months of treatment, MB: Months in bottle. Different letters indicate statistically significant differences $(p<0.05)$ between values.

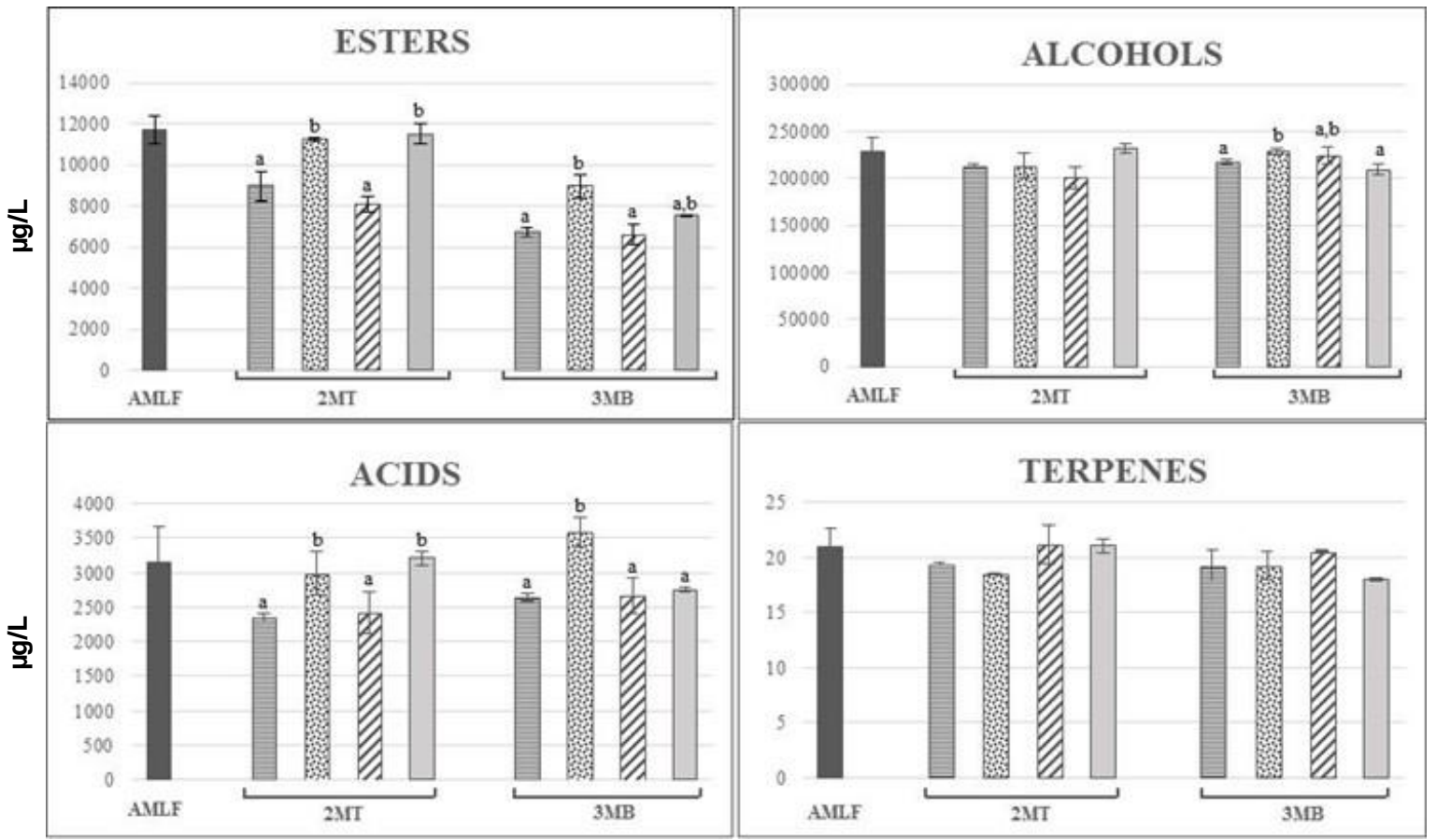

Figure 4. Evolution of the volatile compounds during the treatment and bottle storage in Carménère wines fermented with Uvaferm $\mathrm{HPS}^{\circledR}$. Results expressed in $\mu \mathrm{g} / \mathrm{L} \pm \mathrm{SD}$. Control after malolactic fermentation; 目 Control; 回 SIDY, $\square \mathrm{CW}, \square$ YA. Columns with different letters indicate statistically significant differences $(p<0.05)$ between the different treatments. 2MT: two months of treatment, 3MB: three months in bottle. 
In the case of wines fermented with Uvaferm HPS ${ }^{\circledR}$, those wines treated with SIDY1 and YA had higher concentrations, after the 2MT period, of the esters from all the chemical groups compared to the control, with the exception of isoamyl esters, where only the wines treated with SIDY1 had higher content than the control wines. These differences were maintained after the 3MB period but only in the case of SIDY1. However, it is important to note that the wines treated with YA also had a higher total isoamyl ester than the controls. Another remarkable fact is the significant increase of fatty acids after 2MT in wines treated with SIDY1 and YA, which had also been observed in the wines fermented with Lalvin $\mathrm{EC} 1118^{\circledR}$ yeast but only in the wines treated with CW. Some authors have postulated that the higher levels of fatty acids in wines aged with lees are related to the release of these compounds from cell walls during yeast autolysis [38]. Moreover, these results are in accordance with some studies that found a higher content of some fatty acids using ageing treatment with YDs $[3,39,40]$; for this reason it is possible that the same effect as the ageing on natural lees could have occurred with the use of these products. Other authors have identified fatty acids and their ethyl and methyl esters in several YDs extracts [41], explaining that the great majority seemed to be produced by Maillard reactions, possibly during the thermal processing of these products from yeast sugars and amino acids and/or peptides. In addition, after the $3 \mathrm{MB}$ period, the treatment with SIDY1 accounted for the highest concentration of volatile fatty acids. As occurred in the wines fermented with Lalvin $\mathrm{EC} 1118^{\circledR}$ yeast strain, no significant differences between the control and treated wines were observed in the total alcohols after the 2MT period. However, the treatment with YA produced wines with higher amounts of 3-methyl-1-butanol and octanol than control wines (see the Table 3). Similar results for the total content of fatty acids were found for total alcohols after the 3MB period, showing that the wines treated with SIDY1 had a higher total content than the control and the other treated wines. Conversely to what was observed in the wines fermented with LalvinEC $1118^{\circledR}$, after the $3 \mathrm{MB}$ period, the terpenes group did not change considerably.

The adsorption or enhancing effect of the addition of the same YDs on red wines was mentioned by some authors during the ageing and bottle storage periods [3,40]. Rodríguez-Bencomo et al. [40] explained that, in general, the interactions between YDs and some volatile compounds disappeared during bottling, mainly due to the fact that binding between these products and volatile compounds could be reversible and the latter could be released again into the wine. Del Barrio-Galán et al. [3] also observed an interaction effect between the YDs used and volatile compounds of red wines, but an increase of some of these compounds was also found.

The global balance points out that the wines fermented with Lalvin EC1118 ${ }^{\circledR}$ presented a higher total amount of volatile compounds during $2 \mathrm{MT}$ and after $3 \mathrm{MB}$ compared with the control wines. However, when fermented with Uvaferm $\mathrm{HPS}^{\circledR}$, after $2 \mathrm{MT}$ the treatment with $\mathrm{CW}$ decreased the total amount of volatile compounds and after 3MB the wines treated with YA also had decreased amounts.

Therefore, it seems clear that their effect on the volatile compounds depends on the type of product used, because the soluble colloids from yeasts (especially polysaccharides) can affect the perception of aroma substances in opposite ways, either reducing or increasing their volatility, and could be influenced by the multiple factors mentioned above.

\subsection{Effect of YDs on the Wine Astringency}

Figure 5 shows the percentages of dominance over time for the astringency of the different treatments. For the different wines studied, fermented with either Lalvin EC1118 ${ }^{\circledR}$ or Uvaferm HPS ${ }^{\circledR}$, it can be seen that the control wines had greater dominance and persistence of astringency at the end of the test with respect to the wines treated with YDs.

Figure 6 shows the duration and the final time of the astringency perception. Statistically significant differences were found for both wines fermented with Lalvin EC1118 ${ }^{\circledR}$ and wines fermented with Uvaferm HPS ${ }^{\circledR}$. The control wines obtained a longer duration and greater final time of astringency perception. The time differences in the astringency perception are well correlated with the lower concentrations of polysaccharides present in the control samples, and therefore a greater perception 
of the astringency over time is also present. No clear difference was observed between the different YDs applied.
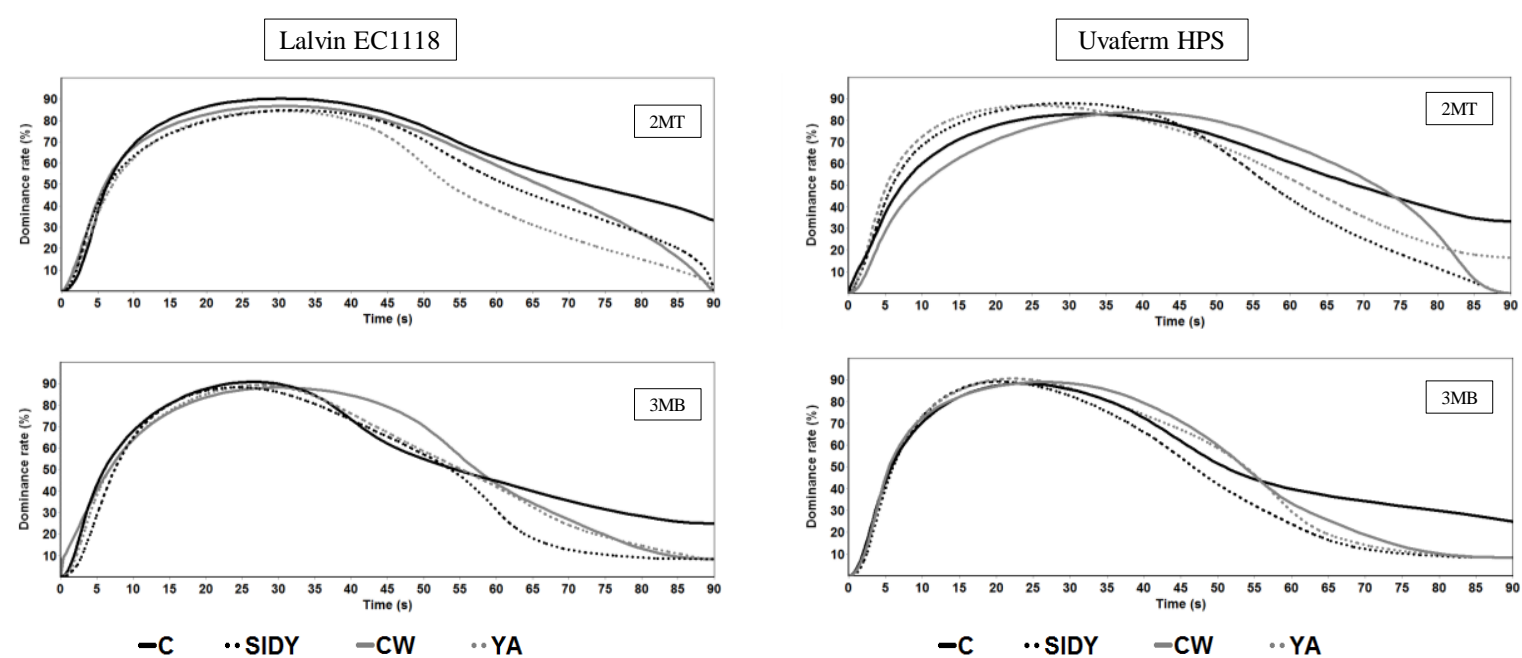

Figure 5. Time intensity profiles of astringency evaluated in the different red wines studied. (s): seconds; $(\%)$ : percentage of dominance rate.
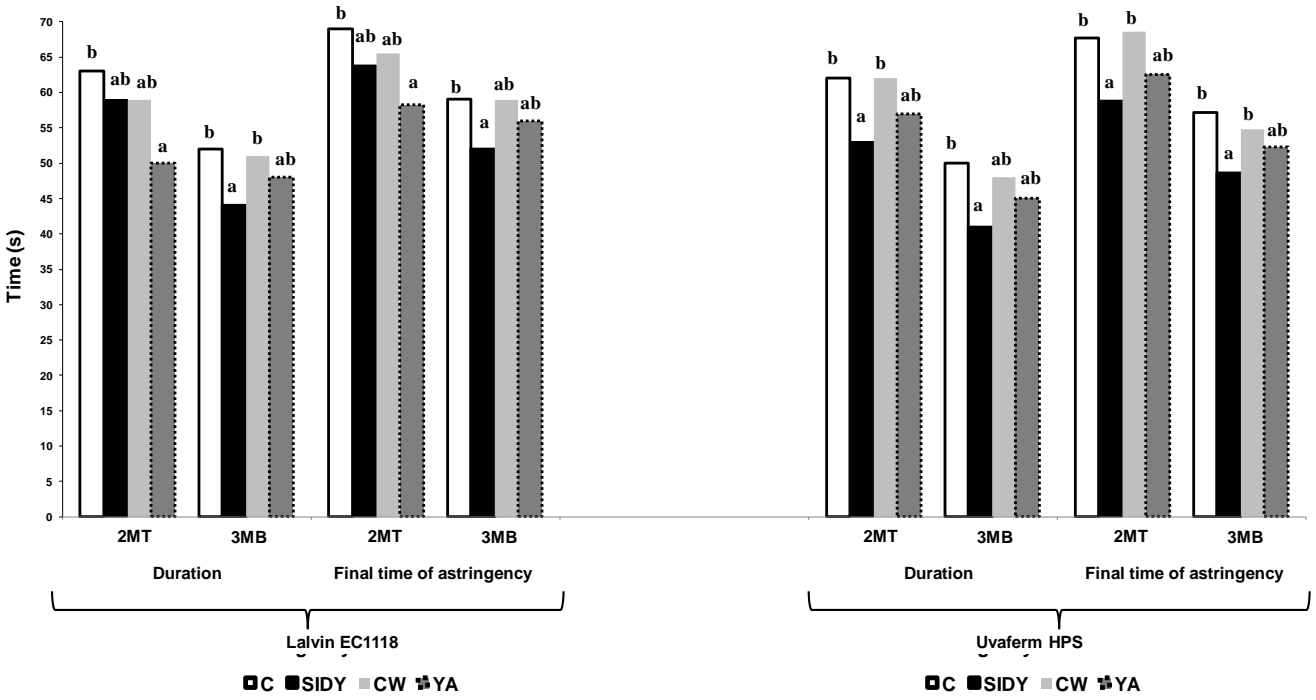

Figure 6. Duration and final time of astringency evaluated in seconds. Columns with different letters indicate statistically significant differences $(p<0.05)$ between the treatments.

Although, in general, the treatments with different YDs did not produce a significant modification of the phenolic compounds content, they had a significant effect on the decrease of the duration of the astringency sensation of the red wines studied. This could be due to the interaction of the polysaccharides released by the YDs and the phenolic compounds of the wine, forming macromolecular structures that remain as stable colloids in wine, as has already been described in some studies [42,43]. According to these studies, polysaccharides and mannoproteins could prevent the tannins' self-aggregation, forming more stable aggregates that prevent their polymerization and subsequent precipitation. This could contribute to maintaining the lubrication of the oral cavity, decreasing the sensation of dryness and roughness that characterize the astringency attribute. 


\section{Materials and Methods}

\subsection{Winemaking and Experimental Design}

The study was carried out on a Carménère wine variety of the 2015 vintage. The wine was made in the Popeta winery, located in the Maipo Valley region of Chile ( $34^{\circ} 27^{\prime} 3,35^{\prime \prime}$ (S) and $70^{\circ} 46^{\prime} 42$, $\left.17^{\prime \prime}(\mathrm{W})\right)$. The alcoholic fermentation was carried out in 300-hL stainless steel tanks. The vinification processes were carried out under the routine work conditions established by the winery.

The same volume of must from the same batch of red grapes, located in the same sector of the vineyard, was fermented with two different yeast strains. One batch was inoculated with $20 \mathrm{~g} / \mathrm{hL}$ of Lalvin EC1118 ${ }^{\circledR}$ Saccharomyces cerevisiae bayanus and the other batch with $20 \mathrm{~g} / \mathrm{hL}$ of Uvaferm HPS ${ }^{\circledR}$ Saccharomyces cerevisiae. Both yeast strains were supplied by Lallemand-Sudamérica. The alcoholic fermentation was carried out at a controlled temperature between $25^{\circ} \mathrm{C}$ and $28^{\circ} \mathrm{C}$. The malolactic fermentation was carried out spontaneously in the winery. The classical ooenological parameters after malolactic fermentation are detailed in Table 4.

Table 4. Classic oenological parameters of red wines after malolactic fermentation.

\begin{tabular}{ccc}
\hline & Lalvin EC1118 $^{\circledR}$ & Uvaferm HPS $^{\circledR}$ \\
\hline Alcoholic degree (vol \%) & 13.8 & 13.7 \\
pH & 3.91 & 3.82 \\
$\mathrm{AT}(\mathrm{g} / \mathrm{L})$ & 3.2 & 3.4 \\
$\mathrm{AV}(\mathrm{g} / \mathrm{L})$ & 0.41 & 0.38 \\
\hline
\end{tabular}

TA: Total acidity expressed in $\mathrm{g} / \mathrm{L}$ of sulphuric acid. VA: Volatile acidity, expressed in $\mathrm{g} / \mathrm{L}$ of acetic acid.

The free $\mathrm{SO}_{2}$ was adjusted to $30 \mathrm{mg} / \mathrm{L}$ after malolactic fermentation was completed and the two different batches of fermented wines were divided into food-grade plastic tanks where different ageing treatments were applied in duplicate (eight tanks $\times 25 \mathrm{~L}$ ).

The different treatments were applied after malolactic fermentation (AMLF): control wines (wines without any YDs applied (C)); wines treated with a commercial specific inactive dry yeast SIDY (SIDY) named Optilees ${ }^{\circledR}$; wines treated with two new experimental YDs based in a cell-wall fraction $(\mathrm{CW})$ and a yeast autolysate (YA). The treatments were performed in an underground cellar with a constant temperature $\left(15 \pm 3{ }^{\circ} \mathrm{C}\right)$.

The YDs were supplied by Lallemand-South America and were applied in a medium-high dosage $(30 \mathrm{~g} / \mathrm{hL})$ based on the technical recommendations range $(20-40 \mathrm{~g} / \mathrm{hL})$. The treatments lasted two months; a weekly "batonnage" was performed during the first month of treatment, and one every two weeks during the second month. After the treatment period the wines were filtered (without clarification treatment) with a cellulose plate filter and bottled, and were stored at a constant temperature (15 \pm $3^{\circ} \mathrm{C}$ ) during three months in bottle.

\subsection{Reagents and Standards}

The standards of gallic, protocatechuic, caffeic, syringic, $p$-coumaric, ferulic, ellagic and caftaric acids, tyrosol, thyptophol, quercetin, myricetin, astilbin, (+)-catechin and (-)-epicatechin, dextrans, and pectins were purchased from Sigma-Aldrich Chemical Co. (St. Louis, MO, USA). Polyethylene membranes of $0.45 \mu \mathrm{m}$ and $0.22 \mu \mathrm{m}$ pore size were acquired from EMD Millipore (Billerica, MA, USA). Sodium sulphate (anhydrous), potassium metabisulfite, vanillin (99\%), ethyl acetate, diethyl ether, sodium hydroxide, acetic acid, formic acid, sulphuric acid, ethanol, hydrochloric acid and high-performance liquid chromatography (HPLC)-grade acetonitrile, methanol, and ammonium formate were purchased from Merck (Darmstadt, Germany). All the reagents were of analytical grade or higher. 


\subsection{Analytical Methods}

The classical ooenological parameters were analysed according to the official methods established by the OIV (2015) [44]. The extraction, determination, and quantification (mg/L) of polysaccharides according to their molecular weight was carried out following the methodology described by Ayestarán et al. [45] using a High-Performance Size Exclusion Chromatography-Refractive Index Detector (HPSEC-RID).

Total polyphenol index (TPI), total anthocyanins (expressed in $\mathrm{mg} / \mathrm{L}$ of malvidin-3-glucoside), and total tannins (expressed in $\mathrm{g} / \mathrm{L}$ of catechin) were analysed according to the methods established by Ribéreau-Gayon et al. [46]. The HPLC-DAD low molecular weight phenolic compounds extraction, determination, and quantification were carried out according to the method proposed by Peña-Neira et al. [47].

Colour intensity and tonality were analysed according to Glories (1984) [48]. CIELab parameters were calculated according to the MSCV ${ }^{\circledR}$ method (Simplified method to determine the colour of wines) developed by the colour group laboratory of the University of La Rioja (Spain) [49].

All the wines were analysed after two months of treatment (2MT) and after three months of bottle storage (3MB). The analysis of volatile compounds was done employing the headspace solid phase micro-extraction method described in Úbeda et al. [50] using 4-methyl-2-pentanol $\left(0.75 \mathrm{mg} \mathrm{L}^{-1}\right)$ as internal standard. After that, gas chromatography analysis was carried out using a 7890B Agilent GC system coupled to a quadrupole mass spectrometer Agilent 5977 inert (Agilent Technologies, Palo Alto, CA, USA). The conditions employed were the same as in Ubeda et al. [50].

The sensory analysis was carried out by 14 trained panellists belonging to the Department of Agro-Industry and Enology of the Agronomical Sciences of the Chile University. The evaluation of the different wines was carried out using FIZZ software (Biosystemes, Dijon, France) and according to the conditions established in [9]. The methodologies used to determine the threshold of astringency perception and to train the panellists in the evaluation and characterization of the astringency attribute are described in Medel-Marabolí et al. [51]. This software is a visual tool that allows one to use different sensory analysis methodologies, automation, and data and processing collection [52]. The methodology used was "Time Intensity", which is a dynamic sensory analysis technique that allows one to observe the progression of a specific sensory attribute over time [53]. In this study, the astringency was evaluated. The intensity of the astringency was evaluated using a $15-\mathrm{cm}$ unstructured scale, where the " 0 value" corresponded with the absence of astringency perception and the " 15 value" with the maximum intensity of astringency perception. The time evaluation established for each wine was $90 \mathrm{~s}$.

This methodology can be very useful to study the temporal perception of a specific sensory attribute in wine. Generally, according to the literature consulted, the "Time Intensity" methodology is carried out on a small number of attributes or with a limited number of products, where only one attribute is evaluated [53].

\subsection{Statistical Analysis}

The statistical analysis was carried out with the statistical program "InfoSat version 2012p" (FCA-National University of Córdoba, Córdoba, Argentina). Data were analysed using the one-way analysis of variance (ANOVA) followed by the LSD-Fisher test, which determined statistically significant differences between the means with a level of significance of $95 \%(p<0.05)$. The different yeast strains were treated as two independent groups.

\section{Conclusions}

The addition of the YDs produced an increase in the different polysaccharidic fractions and the total polysaccharide content, and it was YA that released the highest amounts after the treatment period, regardless of the fermentation yeast used. 
In general, the addition of YDs did not produce a clear trend in the phenolic content of the wines studied, revealing two different effects: adsorption and/or protective of phenolic compounds. In the same way, the results obtained for the colour of wines were quite ambiguous and only in certain cases did we observe an improvement in the red wine's colour, probably due to the different composition of the YDs, the wine matrix, and the harvest effect.

Two different effects were found in the red wine composition: an intensification of the most volatile compound groups, or a lower decrease of these compounds after the treatment period, mainly after the bottle storage period. However, although the same YDs treatments were used for both types of fermented wines, the results were not always the same. This may be due to the importance of the yeast strain used during the fermentation process, because the initial concentration of the evaluated volatile compounds depends on the fermentation yeast strain.

In general, the addition of different YDs into red wines in the post-fermentation process produced a significant decrease in their astringency perception, mainly due to a lower duration of this attribute in the wines treated with these YDs. This could be correlated with the higher amounts of polysaccharides released by these products, producing wines with a better mouthfeel that are thus more attractive to the wine consumer.

Based on these results, and due to the scarce scientific studies considering the great variety of YDs (commercial and in development), more research should be carried out in this area in order to obtain a better understanding of the action mechanisms of these products and their effect on the sensory profile.

Author Contributions: R.D.B.-G. designed the work, performed the experiments and the physicochemical and sensory analysis sessions of the wines, and performed the statistical analysis of the data; M.G. performed the analyses of total polyphenols and tannins and contributed to the interpretation, writing and discussion of the results obtained; C.U. performed the volatile analysis and contributed to the interpretation, writing and discussion of the results obtained; M.M.-M. performed and supervised the sensory analysis sessions, and wrote and discussed the sensory data of astringency; N.S. contributed to design the work, in the technical support about the products and supply the different products used in this study; Á.P.-N. designed the work and contributed to the interpretation of the results, writing, and discussion.

Funding: CONICYT-PAI/Concurso Nacional Inserción en el Sector Productivo, Convocatoria 2014 N $^{\circ} 781403003$, and the projects: FONDECYT 11140275 and FONDECYT N ${ }^{\circ} 1140882$ and FONDEQUIP EQM-130129. Lallemand Inc. Chile y Compañía Limitada.

Acknowledgments: The authors are grateful to the Popeta Winery for providing the wine for the study.

Conflicts of Interest: The authors of University of Chile declare no conflict of interest. Rubén Del Barrio-Galán is associated with Lallemand in a researcher role and Nathalie Sieczkowski is employed at Lallemand as a R\&D Manager. Both authors affiliated with Lallemand have no competing financial interests to declare.

\section{References}

1. Pozo-Bayón, M.A.; Andújar-Ortiz, I.; Moreno-Arribas, M.V. Scientific evidences beyond the application of inactive dry yeast preparations in winemaking. Food Res. Int. 2009, 42, 754-761. [CrossRef]

2. Del Barrio-Galán, R.; Pérez-Magariño, S.; Ortega-Heras, M.; Guadalupe, Z.; Ayestarán, B. Polysaccharide characterization of commercial dry yeast preparations and their effect on white and red wine composition. LWT-Food Sci. Technol. 2012, 48, 215-223.

3. Del Barrio-Galán, R.; Ortega-Heras, M.; Sánchez-Iglesias, M.; Pérez-Magariño, S. Interactions of phenolic and volatile compounds with yeast lees, commercial yeast derivatives and non toasted chips in model solutions and young red wines. Eur. Food Res. Technol. 2012, 234, 231-244. [CrossRef]

4. Guadalupe, Z.; Martínez, L.; Ayestarán, B. Yeast mannoproteins in red winemaking: Effect on polysaccharide, polyphenolic, and colour composition. Am. J. Enol. Viticult. 2010, 61, 191-200.

5. Del Barrio-Galán, R.; Pérez-Magariño, S.; Ortega-Heras, M.; Williams, P.; Doco, T. Effect of ageing on lees and of three different dry yeast derivative products on Verdejo white wine composition and sensorial characteristics. J. Agric. Food Chem. 2011, 59, 12433-12442. [CrossRef]

6. Soubeyrand, V.; Luparia, V.; Williams, P.; Doco, T.; Vernhet, A.; Ortiz-Julien, A. Formation of micelle containing solubilized sterols during rehydration of active dry yeasts improves their fermentation capacity. J. Agric. Food Chem. 2005, 53, 8025-8032. 
7. Feuillat, M.; Gerreau, J. Les nouveaux activateurs de la fermentation alcoolique. Bull. OIV 1996, 789-790, 987-998.

8. Díez, L.; Guadalupe, Z.; Ayestarán, B.; Ruiz-Larrea, F. Effect of yeast mannoproteins and grape polysaccharides on the growth of wine lactic acid and acetic acid bacteria. J. Agric. Food Chem. 2010, 58, 7731-7739.

9. Del Barrio-Galán, R.; Medel-Marabolí, M.; Peña-Neira, A. Effect of different ageing techniques on the polysaccharide and phenolic composition and sensory characteristics of Syrah red wines fermented using different yeast strains. Food Chem. 2015, 179, 116-126. [PubMed]

10. Del Barrio-Galán, R.; Pérez-Magariño, S.; Ortega-Heras, M. Techniques for improving or replacing ageing on lees of oak aged red wines: The effects on polysaccharides and the phenolic composition. Food Chem. 2011, 127, 528-540. [CrossRef]

11. González-Royo, E.; Urtasun, A.; Gil, M.; Kontoudakis, N.; Esteruelas, M.; Fort, F.; Zamora, F. Effect of yeast strain and supplementation with inactive yeast during alcoholic fermentation on wine polysaccharides. Am. J. Enol. Viticult. 2013, 64, 268-273. [CrossRef]

12. Li, S.; Bindon, K.; Bastian, S.; Wilkinson, K. Impact of Commercial Oenotannin and Mannoprotein Products on the Chemical and Sensory Properties of Shiraz Wines Made from Sequentially Harvested Fruit. Foods 2018, 7, 204. [CrossRef]

13. Li, S.; Bindon, K.; Putnam Bastian, S.; Jiranek, V.; Wilkinson, K. Use of Winemaking Supplements to Modify the Composition and Sensory Properties of Shiraz Wine. J. Agric. Food Chem. 2017, 65, 1353-1364. [CrossRef]

14. Mekoue-Nguela, J.; Sieczkowski, N.; Roi, S.; Vernhet, A. Sorption of Grape Proanthocyanidins and wine polyphenols by yeasts, inactivated yeasts, and yeast cell walls. J. Agric. Food Chem. 2015, 63, 660-670. [CrossRef] [PubMed]

15. Mekoue-Nguela, J.; Vernhet, A.; Sieczkowski, N.; Brillouet, J.M. Interactions of Condensed Tannins with Saccharomyces cerevisiae Yeast Cells and Cell Walls: Tannin Location by Microscopy. J. Agric. Food Chem. 2015, 63, 7539-7545. [CrossRef] [PubMed]

16. Escot, S.; Feuillat, M.; Dulau, L.; Charpentier, C. Release of polysaccharides by yeast and the influence of released polysaccharides on colour stability and wine astringency. Aust. J. Grape Wine Res. 2001, 7, 153-159. [CrossRef]

17. Francois, J.M.; Alexandre, H.; Granes, D.; Feuillat, M. Vers une meilleure connaissance des produits dérivés de levures. Rev. Oenolog. 2007, 122, 9-12.

18. Guadalupe, Z.; Ayestarán, B. Effect of commercial mannoprotein addition on polysaccharide, polyphenolic, and colour composition in red wines. J. Agric. Food Chem. 2008, 56, 9022-9029. [CrossRef]

19. Loira, I.; Vejarano, R.; Morata, A.; Ricardo-da-Silva, J.M.; Laureano, O.; González, M.C.; Suárez-Lepe, J.A. Effect of Saccharomyces strains on the quality of red wines aged on lees. Food Chem. 2013, 139, 1044-1051. [CrossRef] [PubMed]

20. Rodrigues, A.; Ricardo-Da-Silva, J.M.; Lucas, C.; Laureano, O. Effect of commercial mannoproteins on wine colour and tannins stability. Food Chem. 2012, 131, 907-914. [CrossRef]

21. Chalier, P.; Angot, B.; Delteil, D.; Doco, T.; Gunata, Z. Interactions between aroma compounds and whole mannoprotein isolated from Saccharomyces cerevisiae strains. Food Chem. 2007, 100, 22-30. [CrossRef]

22. Mahadevan, K.; Farmer, L. Key odor impact compounds in three yeast extracts pastes. J. Agric. Food Chem. 2006, 54, 7242-7250. [CrossRef]

23. Rossetti, D.; Bongaerts, J.H.H.; Wantling, E.; Stokes, J.R.; Williamson, A.M. Astringency of tea catechins: More than an oral lubrication tactile percept. Food Hydrocoll. 2009, 23, 1984-1992. [CrossRef]

24. Rossetti, D.; Yakubov, G.E.; Stokes, J.R.; Williamson, A.M.; Fuller, G.G. Interaction of human whole saliva and astringent dietary compounds investigated by interfacial shear rheology. Food Hydrocoll. 2008, 22, 1068-1078. [CrossRef]

25. Bennick, A. Interaction of plant polyphenols with salivary proteins. Crit. Rev. Oral Biol. Med. 2002, 13, 184-196. [CrossRef] [PubMed]

26. Laguna, L.; Bartolomé, B.; Moreno-Arribas, M.V. Mouthfeel perception of wine: Oral physiology, components and instrumental characterization. Trends Food Sci. Technol. 2017, 59, 49-59. [CrossRef]

27. Quijada-Morín, N.; Williams, P.; Rivas-Gonzalo, J.C.; Doco, T.; Escribano-Bailón, M.T. Polyphenolic, polysaccharide and oligosaccharide composition of Tempranillo red wines and their relationship with the perceived astringency. Food Chem. 2014, 154, 44-51. [CrossRef] 
28. Robichaud, J.L.; Noble, A.C. Astringency and bitterness of selected phenolics in wine. J. Sci. Food Agric. 1990, 53, 343-353. [CrossRef]

29. Ishikawa, T.; Noble, A.C. Temporal perception of astringency and sweetness in red wine. Food Qual. Prefer. 1995, 6, 27-33. [CrossRef]

30. Vidal, L.; Antúnez, L.; Giménez, A.; Medina, K.; Boido, E.; Ares, G. Sensory characterization of the astringency of commercial Tannat wines Uruguayan. Food Res. Int. 2017, 102, 425-434. [CrossRef] [PubMed]

31. Del Barrio-Galán, R.; Cáceres-Mella, A.; Medel-Marabolí, M.; Peña-Neira, A. Effect of selected Saccharomyces cerevisiae yeast strains and different ageing techniques on the polysaccharide and polyphenolic composition and sensorial characteristics of Cabernet Sauvignon red wines. J. Sci. Food Agric. 2015, 95, 2132-2144. [CrossRef]

32. González-Royo, E.; Esteruelas, M.; Kontoudakis, N.; Fort, F.; Canals, J.M.; Zamora, F. The effect of supplementation with three commercial inactive dry yeasts on the colour, phenolic compounds, polysaccharides and astringency of a model wine solution and red wine. J. Sci. Food Agric. 2016, 97, 172-181. [CrossRef]

33. Alcalde-Eon, C.; García-Estévez, I.; Puente, V.; Rivas-Gonzalo, J.C.; Escribano-Bailón, M.T. Colour stabilization of red wines. A chemical and colloidal approach. J. Agric. Food Chem. 2014, 62, 6984-6994. [CrossRef]

34. Watrelot, A.A.; Schulz, D.L.; Kennedy, J.A. Wine polysaccharides influence tannin-protein interactions. Food Chem. 2017, 63, 571-579. [CrossRef]

35. Guzmán-Alfeo, M. Manual de Espectrofotometría para Enología, 1st ed.; AMV Editions: Madrid, Spain, 2010; pp. 110-129, ISBN 978-84-96709-54-6.

36. Etievant, P.X. Volatile Compounds in Foods and Beverages, 1st ed.; Henk Maarse: New York, NY, USA, 1991; pp. 483-533.

37. Pineau, N.; Schlich, P.; Cordelle, S.; Mathonnière, C.; Issanchou, S.; Imbert, A.; Rogeaux, M.P.; Etiévant, E.; Köster, E. Temporal Dominance of Sensations: Construction of the TDS curves and comparison with time-intensity. Food Qual. Prefer. 2009, 20, 450-455. [CrossRef]

38. Pueyo, E.; Martínez-Rodríguez, A.; Polo, M.C.; Santa-Marín, G.; Bartolomé, B. Release of lipids during yeast autolysis in a model wine system. J. Agric. Food Chem. 2000, 48, 116-122. [CrossRef]

39. Comuzzo, P.; Tat, L.; Fenzi, D.; Brotto, L.; Battistutta, F.; Zironi, R. Interactions between yeast autolysates and volatile compounds in wine and model solution. Food Chem. 2011, 127, 473-480. [CrossRef]

40. Rodríguez-Bencomo, J.J.; Ortega-Heras, M.; Pérez-Magariño, S. Effect of alternative techniques to ageing on lees and use of non-toasted oak chips in alcoholic fermentation on the aromatic composition of red wine. Eur. Food Res. Technol. 2010, 230, 485-496.

41. Pozo-Bayón, M.Á.; Andújar-Ortiz, I.; Moreno-Arribas, M.V. Volatile profile and potential of inactive dry yeast-based winemaking additives to modify the volatile composition of wines. J. Sci. Food Agric. 2009, 89, 1665-1673. [CrossRef]

42. Mekoue-Nguela, J.; Poncet-Legrand, C.; Sieczkowski, N.; Vernhet, A. Interactions of grape tannins and wine polyphenols with a yeast protein extract, mannoproteins and $\beta$-glucan. Food Chem. 2016, 210, 671-682. [CrossRef]

43. Poncet-Legrand, C.; Doco, T.; Williams, P.; Vernhet, A. Inhibition of grape seed tannin aggregation by wine mannoproteins: Effect of polysaccharide molecular weight. Am. J. Enol. Viticult. 2007, 58, 87-91.

44. OIV (International Organisation of Vine and Wine). Compendium of International Methods of Wine and Must Analysis; OIV: París, France, 2015; Volumes 1 and 2.

45. Ayestarán, B.; Guadalupe, Z.; León, D. Quantification of major grape polysaccharides (Tempranillo v.) released by maceration enzymes during the fermentation process. Anal. Chim. Acta 2004, 513, 29-39.

46. Ribéreau-Gayon, P.; Glories, Y.; Maujean, A.; Dubordieu, D. The Chemistry of Wine Stabilization and Treatments. In Handbook of Enology; John Wiley \& Sons: Chichester, UK, 2006; pp. 1-230.

47. Peña-Neira, A.; Cáceres, A.; Pastenes, A. Low molecular weight phenolic and anthocyanin composition of grape skins from cv. Syrah (Vitis vinifera L.) in the Maipo Valley (Chile): Effect of clusters thinning and vineyard yield. Food Sci. Technol. Int. 2007, 13, 153-158. [CrossRef]

48. Glories, Y. La couleur des vins rouges. 2éme partie. Mesure, origine et interprétation. Connaissance Vigne Vin 1984, 18, 253-271.

49. Ayala, F.; Echávarri, J.F.; Negueruela, A.I. MSCV ${ }^{\circledR}$. 2014. Available online: http://www.unirioja.es/colour/ descargas.shtml (accessed on 29 March 2019). 
50. Úbeda, C.; Del Barrio-Galán, R.; Peña-Neira, A.; Medel-Marabolí, M.; Durán-Guerrero, E. Location effects on the aromatic composition of monovarietal cv. Carignan wines. Am. J. Enol. Vitic. 2017, 68, 390-399.

51. Medel-Marabolí, M.; Romero, J.L.; Obreque-Slier, E.; Contreras, A.; Peña-Neira, Á. Effect of a commercial tannin on the sensorial temporality of astringency. Food Res. Int. 2017, 102, 341-347. [CrossRef] [PubMed]

52. Lawless, H.T.; Heymann, H. Sensory Evaluation of Foods, Principles and Practices; Springer: New York, NY, USA, 2010.

53. Pineau, B.; Barbe, J.C.; Van Leeuwen, C.; Dubourdieu, D. Examples of perceptive interactions involved in specific "Red" and "Black-berry" aromas in red wines. J. Agric. Food Chem. 2009, 57, 3702-3708. [CrossRef]

Sample Availability: Samples of the compounds are not available from the authors.

(C) 2019 by the authors. Licensee MDPI, Basel, Switzerland. This article is an open access article distributed under the terms and conditions of the Creative Commons Attribution (CC BY) license (http://creativecommons.org/licenses/by/4.0/). 